Accepted for publication in Applied Mathematical Modelling (13. Dec. 2017)

The publication is available at Elsevier via https://doi.org/10.1016/j.apm.2017.12.029

\title{
Numerical methods for the stability of time-periodic hybrid time-delay systems with applications
}

\author{
David Lehotzky*,a, Tamas Insperger ${ }^{\mathrm{a}}$, and Gabor Stepan ${ }^{\mathrm{b}}$

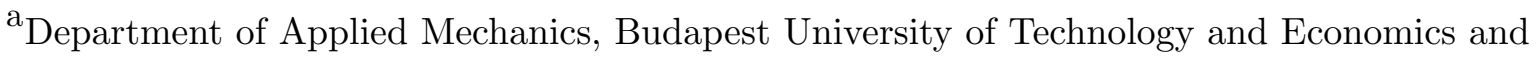 \\ MTA-BME Lendület Human Balancing Research Group, 1111 Budapest, Hungary \\ ${ }^{\mathrm{b}}$ Department of Applied Mechanics, Budapest University of Technology and Economics, Hungary, \\ 1111, Budapest, Muegyetem rkp. 3.
}

\begin{abstract}
This paper extends two numerical methods for the stability analysis of a class of time-periodic hybrid time-delay systems. In particular, the pseudospectral tau and spectral element methods are extended to hybrid systems. The analyzed delay-differential equation involves delayed terms with both continuous and piecewise constant arguments, in other words, it involves delays both without and with zero-order hold, respectively. The analyzed class of hybrid systems can be used to describe time-periodic hereditary processes subjected to digital feedback control. The proposed numerical algorithms are applied to the mathematical models of haptic systems and milling processes subjected to digital feedback control.
\end{abstract}

Keywords: time-delay system; hybrid system; time-periodic system; digital control; haptic system; milling process

\footnotetext{
*Corresponding author

E-mail address: lehotzky@mm.bme.hu
} 


\section{Introduction}

As the importance of delay effects has been realized in several engineering and biological applications, the attention to the analysis of time-delay systems has increased in the recent decades. Time delay is inherently present in machine tool vibrations [1], control systems [2], traffic dynamics [3, human balancing [4], population dynamics [5] or epidemiology [6], just to mention a few examples. The local stability analysis of these time-delay systems provides the primary characteristics of their behavior around stationary states. Consequently, several analytical and numerical methods have been developed for local stability analysis. For instance, the Dsubdivision method [7] gives a closed form solution for the stability boundaries of autonomous delay-differential equations in the space of system parameters. While closed form solutions can be derived for the stability boundaries of most autonomous systems, the stability analysis of time-periodic systems usually requires numerical approximation techniques, especially in the presence of time-delays. The related literature provides many numerical methods for the stability analysis of time-periodic time-delay systems, such as the semi-discretization method [8], full discretization method [9], spectral element method [10] or the pseudospectral collocation method [11, 12], just to mention a few examples.

In digitally controlled mechanical systems (see e.g. [13]), the state variables in the governing equations appear with piecewise constant arguments due to sampled data hold in the feedback loop, which results in a hybrid system. Here, the terms with piecewise constant arguments will be referred as terms with discrete delays. The open-loop system can also incorporate delayed terms, whose arguments are continuous functions of time. In the following, these terms will be referred as terms with continuous delays.

Mathematical models involving both discrete and continuous delays can arise in case of coupled haptic systems (see e.g. [14, 15]), where the haptic device is subjected to both human interaction and digital feedback control. In these models, continuous delay terms are present due to the reaction delay of the human operator, while discrete delay terms are originated from the sampling and actuation scheme of the digital controller. Further examples for hybrid timedelay systems can be taken from the field of machine tool vibrations. Here the continuous delay is present due to the so-called regenerative effect of the cutting process [16], while the discrete delays are originated from the feedback control of the workpiece-tool system and from the feedback loop of the active damper used for the vibration suppression of the cutting tool. In spite of the relevant applications of time-delay systems subjected to digital feedback control, their numerical stability analysis is not well-established in the engineering literature. In this paper, two existing numerical methods, the pseudospectral tau (PT) method and the spectral element (SE) method are extended for the stability analysis of linear hybrid time-periodic time-delay systems.

The extended numerical methods are tested on two engineering applications. First, the mathematical model of a coupled haptic system is analyzed. To our knowledge, stability analysis of coupled haptic systems has been carried out in the literature either with the omission of discrete delay terms or with the substitution of continuous delay terms by continuous nondelayed terms. Therefore, this paper presents the stability analysis of a coupled haptic system considering continuous and discrete delays simultaneously.

The second application is for milling processes subjected to active damping. In the machining literature, such processes have been modeled using continuous, non-delayed feedback terms in the control input. However in practice, active damping is realized using a digital feedback controller which necessitates the incorporation of discrete delay terms in the governing equations. In this paper, the stability analysis of milling processes subjected to active damping is performed with the consideration of discrete delay in the feedback loop. 


\section{The equation under analysis}

In this paper the stability of the dynamical system

$$
\dot{\mathbf{x}}(t)=\mathbf{A}(t) \mathbf{x}(t)+\sum_{a=1}^{m} \mathbf{B}_{a}(t) \mathbf{x}\left(t-\tau_{a}\right)+\sum_{b=0}^{n} \mathbf{C}_{b} \mathbf{x}\left(t_{j}-b \Delta t\right), \quad t \in\left[t_{j}, t_{j+1}\right),
$$

is analyzed with initial condition

$$
\mathbf{x}(\theta)=\mathbf{x}_{0}(\theta), \quad \theta \in[-\tau, 0]
$$

where $j \in \mathbb{N} ; \mathbf{x}:[-\tau, \infty) \rightarrow \mathbb{R}^{s} ; \mathbf{A}, \mathbf{B}_{a}: \mathbb{R} \rightarrow \mathbb{R}^{s \times s} ; \mathbf{C}_{b} \in \mathbb{R}^{s \times s}$ and $\mathbf{A}(t)=\mathbf{A}(t+T)$, $\mathbf{B}_{a}(t)=\mathbf{B}_{a}(t+T) \forall t$, with $T$ being the time-period of the periodic coefficients. The nonzero continuous delays are denoted by $\tau_{\mathrm{a}}>0, a=1,2, \ldots, m$ and the length of time history is $\tau=\max \left(\tau_{1}, \tau_{2}, \ldots, \tau_{m}, n \Delta t\right)$. Equation (1) models the digital feedback control of a timeperiodic system with system delays. The digital control applies state feedback via gain matrices $\mathbf{C}_{b}$. The state is measured at each sampling instant $t_{j}+b \Delta t, j \in \mathbb{N}, b=0,1, \ldots, n$; while the control input is updated at time instants $t_{j}=j \Delta T, j \in \mathbb{N}$, where $\Delta t$ is the sampling period and $\Delta T=v \Delta t$ is the actuation period, with $v \in \mathbb{Z}^{+}$being the number of samples between two control input updates while $\mathbb{Z}^{+}$denotes the set of positive integer numbers. The control input is held constant between the two endpoints of each actuation period in accordance with a zero-order hold. In this paper it is assumed that the calculation of the control input requires the knowledge of the state in $n+1$ consecutive sampling instants from domain $\left[t_{j}-n \Delta t, t_{j}\right]$, therefore the state-feedback terms in (1) are subjected to delays. The employment of past samples aims to cover a broader group of control methods of delayed systems (involving e.g. the finite spectrum assignment [17], Smith predictor [18], act-and-wait [19] and optimal control [20] methods), where not only the most recent samples but past samples or a weighted sum of past samples also contribute to the control law. In (1), $\mathbf{x}\left(t-\tau_{a}\right), a=1,2, \ldots, m$; are terms with continuous delays, while $\mathbf{x}\left(t_{j}-b \Delta t\right), j \in \mathbb{N}, b=0,1, \ldots, n$; are terms with discrete delays. In case of a general choice for $T$ and $\Delta T$ this system is quasi-periodic. In this paper it is assumed that $T / \Delta T=\sigma / \rho$, with $\sigma, \rho \in \mathbb{Z}^{+}$, thus $T_{\mathrm{p}}=\sigma \Delta T=\rho T$ is the principal period of the system. Note that although (1) contains only constant continuous delays (pointwise delays), DDEs involving distributed continuous delay terms can also be approximated by (1) using numerical quadratures as introduced in [21].

The stability of (1) is determined by the characteristic multipliers of the monodromy operator $\mathcal{U}$, which is defined as

$$
\mathbf{x}_{j T_{\mathrm{p}}}=\mathcal{U} \mathbf{x}_{(j-1) T_{\mathrm{p}}}, \quad j=\mathbb{Z}^{+},
$$

where function segment $\mathbf{x}_{t}$ is given by the shift

$$
\mathbf{x}_{t}(\theta)=\mathbf{x}(t+\theta), \quad \theta \in[-\tau, 0] .
$$

Equation (1) is asymptotically stable if and only if all the characteristic multipliers of $\mathcal{U}$ are within the unit circle of the complex plane [22. In the next section, the extension of the PT and the SE methods are presented for the matrix approximations of $\mathcal{U}$. 


\section{$3 \quad$ Numerical stability analysis}

This section details the application of the PT [23] and SE [21] methods for the approximation of the monodromy operator of the hybrid system (1). While the PT method discretizes the operator differential equation form, the SE method is based on the operator equation form of (1). For the approximation of the operator differential equation and the operator equation, both numerical techniques use the method of weighted residuals and the tau method. In this section, the main steps of the approximation schemes are given for the hybrid system (1). The full description of the PT and SE methods for continuous-time (non-hybrid) systems can be found in [23] and [21], respectively.

\subsection{Pseudospectral tau method}

Using function segment $\mathbf{x}_{t}$, (1) can be converted to the operator differential equation form

$$
\dot{\mathbf{x}}_{t}=\mathcal{G}(t) \mathbf{x}_{t}+\mathcal{H} \mathbf{x}_{t_{j}}, \quad t \in\left[t_{j}, t_{j+1}\right),
$$

where operators $\mathcal{G}(t)$ and $\mathcal{H}$ are defined as

$$
\begin{aligned}
(\mathcal{G}(t) \boldsymbol{\psi})(\theta) & = \begin{cases}\mathbf{A}(t) \boldsymbol{\psi}(0)+\sum_{a=1}^{m} \mathbf{B}_{a}(t) \boldsymbol{\psi}\left(-\tau_{a}\right) & \theta=0, \\
\frac{\mathrm{d}}{\mathrm{d} \theta} \boldsymbol{\psi}(\theta) & \theta \in[-\tau, 0),\end{cases} \\
(\mathcal{H} \boldsymbol{\psi})(\theta) & = \begin{cases}\sum_{b=0}^{n} \mathbf{C}_{b} \boldsymbol{\psi}(-b \Delta t) & \theta=0, \\
\mathbf{0} & \theta \in[-\tau, 0),\end{cases}
\end{aligned}
$$

with $\boldsymbol{\psi}:[-\tau, 0] \rightarrow \mathbb{R}^{s}$. The PT method approximates function segment $\mathbf{x}_{t}$ with its Lagrange interpolant $\tilde{\mathbf{x}}_{t}$ as

$$
\tilde{\mathbf{x}}_{t}(\theta)=\sum_{k=1}^{N+1} \phi_{k}(\theta) \tilde{\mathbf{x}}_{t}\left(\theta_{k}\right)
$$

where $\phi_{k}(\theta)$ are the Lagrange base polynomials (see Appendix A with $\left\{\theta_{k}\right\}_{k=1}^{N+1}$ being the point set of interpolation. After the substitution of (7) to (1), the equation is multiplied with test functions $\left\{\psi_{i}(\theta)\right\}_{i=1}^{N}$ and integrated over domain $\theta \in[-\tau, 0]$ according to the standard steps of the weighted residual method. In case of independent test functions, this gives $N$ independent equations and the $(N+1)$-st equation is obtained from the pointwise satisfaction of (5) at $\theta=0$. Consequently, the final form of the approximate system is given by

$$
\mathbf{N} \dot{\mathbf{y}}(t)=\mathbf{M}(t) \mathbf{y}(t)+\mathbf{H y}\left(t_{j}\right), \quad t \in\left[t_{j}, t_{j+1}\right),
$$

where, after coordinate transformation $\zeta=2 \theta / \tau+1$, the sub-matrices of $\mathbf{N}, \mathbf{M}(t), \mathbf{H} \in$ $\mathbb{R}^{s(N+1) \times s(N+1)}$ and the sub-vectors of $\mathbf{y}(t) \in \mathbb{R}^{s(N+1) \times 1}$ are given as

$$
\begin{aligned}
\mathbf{N}_{i, k}= & \begin{cases}\mathbf{I} \sum_{q=1}^{N+1} \phi_{k}\left(\eta_{q}\right) \psi_{i}\left(\eta_{q}\right) w_{q} & i=1,2, \ldots, N ; \\
\mathbf{I} \phi_{k}(1) & i=N+1 ;\end{cases} \\
\mathbf{M}_{i, k}(t) & = \begin{cases}\mathbf{I} \frac{2}{\tau} \sum_{q=1}^{N+1} \sum_{l=1}^{N+1} \phi_{k}^{\prime}\left(\zeta_{l}\right) \phi_{l}\left(\eta_{q}\right) \psi_{i}\left(\eta_{q}\right) w_{q} & i=1,2, \ldots, N ; \\
\mathbf{A}(t) \phi_{k}(1)+\sum_{a=1}^{m} \mathbf{B}_{a}(t) \phi_{k}\left(1-2 \tau_{a} / \tau\right) & i=N+1 ;\end{cases} \\
\mathbf{H}_{i, k} & = \begin{cases}\mathbf{0} & i=1,2, \ldots, N \\
\sum_{b=0}^{n} \mathbf{C}_{b} \phi_{k}(1-2 b \Delta t / \tau) & i=N+1 ;\end{cases} \\
\mathbf{y}_{k}(t) & =\tilde{\mathbf{x}}_{t}\left(\tau\left(\zeta_{k}-1\right) / 2\right),
\end{aligned}
$$


where $\left\{\eta_{q}\right\}_{q=1}^{N+1}$ is the quadrature point set and $w_{q}$ are the weights used for numerical integration. During computations, the point set of interpolation $\left\{\zeta_{k}\right\}_{k=1}^{N+1}$ is chosen to be the Chebyshev points of second kind (see Appendix B), while the quadrature points $\left\{\eta_{q}\right\}_{q=1}^{N+1}$ are set to be the Legendre-Gauss-Lobatto points (see Appendix C). The test functions are selected as the set $\left\{\psi_{i}(\zeta)\right\}_{i=1}^{N}$ of Legendre polynomials up to degree $N-1$ (see Appendix D. Note that the PT method is not restricted to the above mentioned sets of interpolation points, quadrature points and test functions. Other sets of points (e.g. the Clenshaw-Curtis [24] points) can be used alternatively for integration and interpolation as long as they are convergent on continuous functions. The set of test functions is required to span an $N$-dimensional space in the space of interpolation functions.

The PT method approximates the hybrid time-periodic DDE (1) by the hybrid time-periodic ordinary differential equation (ODE) (8). In order to obtain an approximation for the monodromy operator, (8) is further approximated using a piecewise constant approximation of $\mathbf{M}(t)$. In particular, the actuation period $\Delta T$ is split onto $\tilde{v}$ intervals and $\mathbf{M}(t)$ is averaged as

$$
\tilde{\mathbf{M}}_{j, u}=\frac{1}{\Delta \tilde{t}} \int_{0}^{\Delta \tilde{t}} \mathbf{M}((j \tilde{v}+u-1) \Delta \tilde{t}+t) \mathrm{d} t
$$

where $\Delta \tilde{t}=\Delta T / \tilde{v}$ with $\tilde{v} \in \mathbb{Z}^{+}$and $u=1,2, \ldots, \tilde{v} ; j=0,1, \ldots, \sigma-1$. This way, the timeperiodic ODE (8) is approximated on $t \in\left[0, T_{\mathrm{p}}\right)$ with the series of autonomous ODEs

$$
\begin{gathered}
\mathbf{N} \dot{\tilde{\mathbf{y}}}^{j, u}(t)=\tilde{\mathbf{M}}_{j, u} \tilde{\mathbf{y}}^{j, u}(t)+\mathbf{H} \tilde{\mathbf{y}}^{j, 0}(j \tilde{v} \Delta \tilde{t}), \quad t \in[(j \tilde{v}+u-1) \Delta \tilde{t},(j \tilde{v}+u) \Delta \tilde{t}), \\
\tilde{\mathbf{y}}^{j, u}((j \tilde{v}+u-1) \Delta \tilde{t})=\tilde{\mathbf{y}}^{j, u-1}((j \tilde{v}+u-1) \Delta \tilde{t}),
\end{gathered}
$$

with $j=0,1, \ldots, \sigma-1$. Here, $\tilde{\mathbf{y}}^{j, u}(t)$ is the approximation of $\mathbf{y}(t)$ in the domain $t \in$ $[(j \tilde{v}+u-1) \Delta \tilde{t},(j \tilde{v}+u) \Delta \tilde{t})$. Equation (11) results in the discrete mapping

$$
\mathbf{Y}_{j+1}=\mathbf{\Phi}_{j} \mathbf{Y}_{j}, \quad j=1,2, \ldots, \sigma-1
$$

between the two endpoints of actuation periods (that is between time instants $t_{j}$ and $t_{j+1}$ ), where

$$
\mathbf{Y}_{j}=\tilde{\mathbf{y}}^{j, 0}(j \tilde{v} \Delta \tilde{t}), \quad \boldsymbol{\Phi}_{j}=\boldsymbol{\alpha}_{j}+\boldsymbol{\beta}_{j, \tilde{v}} \mathbf{N}^{-1} \mathbf{H},
$$

with

$$
\boldsymbol{\alpha}_{j}=\mathrm{e}^{\tilde{\mathbf{G}}_{j, \tilde{v}}} \mathrm{e}^{\tilde{\mathbf{G}}_{j, \tilde{v}-1}} \cdots \mathrm{e}^{\tilde{\mathbf{G}}_{j, 1}},
$$

and $\boldsymbol{\beta}_{j, \tilde{v}}$ is defined by the recurrence relation

$$
\boldsymbol{\beta}_{j, u}=\mathrm{e}^{\tilde{\mathbf{G}}_{j, u}} \boldsymbol{\beta}_{j, u-1}+\int_{0}^{\Delta \tilde{t}} \mathrm{e}^{\tilde{\mathbf{G}}_{j, u}(1-t / \Delta \tilde{t})} \mathrm{d} t, \quad u=1,2, \ldots, \tilde{v}
$$

with

$$
\boldsymbol{\beta}_{j, 0}=\mathbf{0}, \quad \tilde{\mathbf{G}}_{j, u}=\Delta \tilde{t} \mathbf{N}^{-1} \tilde{\mathbf{M}}_{j, u} .
$$

Note that when $\tilde{\mathbf{G}}_{j, u}$ is invertible, one gets

$$
\int_{0}^{\Delta \tilde{t}} \mathrm{e}^{\tilde{\mathbf{G}}_{j, u}(1-t / \Delta \tilde{t})} \mathrm{d} t=\Delta \tilde{t}\left(\mathrm{e}^{\tilde{\mathbf{G}}_{j, u}}-\mathbf{I}\right) \tilde{\mathbf{G}}_{j, u}^{-1}
$$

Finally, the matrix approximation of the monodromy operator is

$$
\mathbf{U}=\boldsymbol{\Phi}_{\sigma-1} \boldsymbol{\Phi}_{\sigma-2} \cdots \boldsymbol{\Phi}_{0} .
$$




\section{$3.2 \quad$ Spectral element method}

The SE method converts the original problem (1) to the operator equation

$$
(\mathcal{A} \mathbf{z})(t)=\mathbf{0}, \quad t \in\left[0, T_{\mathrm{p}}\right)
$$

where operator $\mathcal{A}$ is defined as

$$
(\mathcal{A} \mathbf{z})(t)=\dot{\mathbf{z}}(t)-\mathbf{A}(t) \mathbf{z}(t)-\sum_{a=1}^{m} \mathbf{B}_{a}(t) \mathbf{z}\left(t-\tau_{a}\right)-\sum_{b=0}^{n} \mathbf{C}_{b} \mathbf{z}\left(t_{j}-b \Delta t\right), \quad t \in\left[t_{j}, t_{j+1}\right)
$$

$j=0,1, \ldots, \sigma-1$. The domain $t \in\left[-\Gamma T_{\mathrm{p}}, T_{\mathrm{p}}\right)$ of $\mathbf{z}(t)$ is then split to $(\Gamma+1) E \rho$ number of equidistant segments, where

$$
\Gamma= \begin{cases}\text { floor }\left(\frac{\max \left(\tau, T_{\mathrm{p}}\right)}{T_{\mathrm{p}}}\right) & \text { if } \max \left(\tau, T_{\mathrm{p}}\right) \bmod T_{\mathrm{p}}=0 \\ \text { floor }\left(\frac{\max \left(\tau, T_{\mathrm{p}}\right)}{T_{\mathrm{p}}}\right)+1 & \text { otherwise }\end{cases}
$$

$E$ is the number of segments within time period $T$ and the modulo operation is defined according to

$$
a \bmod b=a-b \text { floor }(a / b) .
$$

With the introduction of equidistant segments (19) transforms to

$$
\begin{aligned}
\left(\mathcal{S}^{l} \mathbf{z}_{l}\right)(t)-\sum_{a=1}^{m}\left(\mathcal{Q}^{l, a} \mathbf{z}_{l-r_{a}-1}\right)(t)- & \sum_{a=1}^{m}\left(\mathcal{R}^{l, a} \mathbf{z}_{l-r_{a}}\right)(t) \\
& -\sum_{b=0}^{n} \sum_{j=j_{l-1}}^{j_{l}}\left(\mathcal{P}^{l, j, b} \mathbf{z}_{d_{j v-b}}\right)(t)=\mathbf{0}, \quad t \in[(l-1) h, l h),
\end{aligned}
$$

where $l=1,2, \ldots, E \rho$; and solution segment (or element) $\mathbf{z}_{l}$ denotes the solution $\mathbf{z}(t)$ over domain $t \in[(l-1) h, l h)$, with $h=T / E$ being the element length. Boundary conditions

$$
\mathbf{z}_{l+1}(l h)=\mathbf{z}_{l}(l h), \quad l=-\Gamma E \rho+1,-\Gamma E \rho+2, \ldots, E \rho-1 ;
$$

provide the continuity between adjacent elements. Operators related to the continuous terms are defined as

$$
\begin{aligned}
\left(\mathcal{S}^{l} \mathbf{z}_{l}\right)(t) & = \begin{cases}\dot{\mathbf{z}}_{l}(t)-\mathbf{A}(t) \mathbf{z}_{l}(t) & \text { if } t \in[(l-1) h, l h), \\
\mathbf{0} & \text { otherwise }\end{cases} \\
\left(\mathcal{Q}^{l, a} \mathbf{z}_{l-r_{a}-1}\right)(t) & = \begin{cases}\mathbf{B}_{a}(t) \mathbf{z}_{l-r_{a}-1}\left(t-\tau_{a}\right) & \text { if } t \in\left[(l-1) h,(l-1) h+\vartheta_{a}\right), \\
\mathbf{0} & \text { otherwise, }\end{cases} \\
\left(\mathcal{R}^{l, a} \mathbf{z}_{l-r_{a}}\right)(t) & = \begin{cases}\mathbf{B}_{a}(t) \mathbf{z}_{l-r_{a}}\left(t-\tau_{a}\right) & \text { if } t \in\left[(l-1) h+\vartheta_{a}, l h\right), \\
\mathbf{0} & \text { otherwise }\end{cases}
\end{aligned}
$$

while operators related to the hybrid terms are

$$
\left(\mathcal{P}^{l, j, b} \mathbf{z}_{d_{j v-b}}\right)(t)= \begin{cases}\mathbf{C}_{b} \mathbf{z}_{d_{j v-b}}(j \Delta T-b \Delta t) & \text { if } t \in\left[\kappa_{1}^{l, j}, \kappa_{2}^{l, j}\right) \\ \mathbf{0} & \text { otherwise }\end{cases}
$$


where for $\Delta T / h \leq 1$

$$
\begin{aligned}
& \kappa_{1}^{l, j}= \begin{cases}(l-1) h & j=j_{l-1}, \\
(l-1) h+\Delta T\left(j-j_{l-1}\right)-\gamma_{l-1} & j_{l-1}<j<j_{l}, \\
l h-\gamma_{l} & j=j_{l},\end{cases} \\
& \kappa_{2}^{l, j}= \begin{cases}(l-1) h+\Delta T\left(j+1-j_{l-1}\right)-\gamma_{l-1} & j_{l-1} \leq j<j_{l}, \\
l h & j=j_{l},\end{cases}
\end{aligned}
$$

and for $\Delta T / h>1$

$$
\begin{aligned}
& \kappa_{1}^{l, j}= \begin{cases}(l-1) h & \text { if } j=j_{l} \text { and } \gamma_{l} \geq h, \\
(l-1) h & \text { if } j=j_{l-1} \text { and } \gamma_{l}<h, \\
j_{l} \Delta T & \text { if } j=j_{l} \text { and } \gamma_{l}<h,\end{cases} \\
& \kappa_{2}^{l, j}= \begin{cases}l h & \text { if } j=j_{l}, \\
j_{l} \Delta T & \text { if } j=j_{j-1},\end{cases}
\end{aligned}
$$

with

$$
\begin{aligned}
r_{a}= & \left\{\begin{array}{ll}
\text { floor }\left(\frac{\tau_{a}}{h}\right)-1 & \text { if } \tau_{a} \bmod h=0, \\
\text { floor }\left(\frac{\tau_{a}}{h}\right) & \text { otherwise, }
\end{array} \quad \vartheta_{a}= \begin{cases}h & \text { if } \tau_{a} \bmod h=0, \\
\tau_{a} \bmod h & \text { otherwise }\end{cases} \right. \\
& j_{l}=\text { floor }\left(\frac{l h}{\Delta T}\right), \quad \gamma_{l}=(l h) \bmod \Delta T, \quad d_{e}=\text { floor }\left(\frac{e \Delta t}{h}\right)+1 .
\end{aligned}
$$

After applying the elementwise coordinate transformation

$$
\zeta^{l}=\frac{2(t-(l-1) h)}{h}-1
$$

and dropping index $l$ immediately (since $\zeta^{l} \in[-1,1), \forall l$ ), equation $23-(26)$ is transformed as follows

$$
\begin{aligned}
\left(\mathcal{S}^{l} \mathbf{z}_{l}\right)(\zeta)-\sum_{a=1}^{m}\left(\mathcal{Q}^{l, a} \mathbf{z}_{l-r_{a}-1}\right)(\zeta)-\sum_{a=1}^{m}\left(\mathcal{R}^{l, a} \mathbf{z}_{l-r_{a}}\right)(\zeta) & \\
& -\sum_{b=0}^{n} \sum_{j=j_{l-1}}^{j_{l}}\left(\mathcal{P}^{l, j, b} \mathbf{z}_{d_{j v-b}}\right)(\zeta)=\mathbf{0}, \quad \zeta \in[-1,1),
\end{aligned}
$$

$l=1, \ldots, E \rho$

$$
\begin{gathered}
\mathbf{z}_{l+1}(-1)=\mathbf{z}_{l}(1), \quad l=-\Gamma E \rho+1,-\Gamma E \rho+2, \ldots, E \rho-1 ; \\
\left(\mathcal{S}^{l} \mathbf{z}_{l}\right)(\zeta)= \begin{cases}\frac{2}{h} \mathbf{z}_{l}^{\prime}(\zeta)-\mathbf{A}\left(\frac{h}{2}(\zeta+1)+h(l-1)\right) \mathbf{z}_{l}(\zeta) & \text { if } \zeta \in[-1,1), \\
\mathbf{0} & \text { otherwise, }\end{cases} \\
\left(\mathcal{Q}^{l, a} \mathbf{z}_{l-r_{a}-1}\right)(\zeta)= \begin{cases}\mathbf{B}_{a}\left(\frac{h}{2}(\zeta+1)+h(l-1)\right) \mathbf{z}_{l-r_{a}-1}\left(\zeta+2-\tilde{\vartheta}_{a}\right) & \text { if } \zeta \in\left[-1,-1+\tilde{\vartheta}_{a}\right), \\
\mathbf{0} & \text { otherwise, }\end{cases} \\
\left(\mathcal{R}^{l, a} \mathbf{z}_{l-r_{a}}\right)(\zeta)= \begin{cases}\mathbf{B}_{a}\left(\frac{h}{2}(\zeta+1)+h(l-1)\right) \mathbf{z}_{l-r_{a}}\left(\zeta-\tilde{\vartheta}_{a}\right) & \text { if } \zeta \in\left[-1+\tilde{\vartheta}_{a}, 1\right), \\
\mathbf{0} & \text { otherwise, }\end{cases} \\
\left(\mathcal{P}^{l, j, b} \mathbf{z}_{d_{j v-b}}\right)(\zeta)= \begin{cases}\mathbf{C}_{b} \mathbf{z}_{d_{j v-b}}\left(1-\tilde{\nu}_{j v-b}\right) & \text { if } \zeta \in\left[\tilde{\kappa}_{1}^{l, j}, \tilde{\kappa}_{2}^{l, j}\right), \\
\mathbf{0} & \text { otherwise, }\end{cases}
\end{gathered}
$$


respectively, where for $\Delta T / h \leq 1$

$$
\begin{aligned}
& \tilde{\kappa}_{1}^{l, j}= \begin{cases}-1 & \text { if } j=j_{l-1}, \\
-1+\Delta \tilde{T}\left(j-j_{l-1}\right)-\tilde{\gamma}_{l-1} & \text { if } j_{l-1}<j<j_{l}, \\
1-\tilde{\gamma}_{l} & \text { if } j=j_{l},\end{cases} \\
& \tilde{\kappa}_{2}^{l, j}= \begin{cases}-1+\Delta \tilde{T}\left(j+1-j_{l-1}\right)-\tilde{\gamma}_{l-1} & \text { if } j_{l-1} \leq j<j_{l}, \\
1 & \text { if } j=j_{l}\end{cases}
\end{aligned}
$$

and for $\Delta T / h>1$

$$
\begin{aligned}
& \tilde{\kappa}_{1}^{l, j}= \begin{cases}-1 & \text { if } j=j_{l} \text { and } \gamma_{l} \geq h, \\
-1 & \text { if } j=j_{l-1} \text { and } \gamma_{l}<h, \\
1-\tilde{\gamma}_{l} & \text { if } j=j_{l} \text { and } \gamma_{l}<h,\end{cases} \\
& \tilde{\kappa}_{2}^{l, j}= \begin{cases}1 & \text { if } j=j_{l}, \\
1-\tilde{\gamma}_{l} & \text { if } j=j_{j-1},\end{cases}
\end{aligned}
$$

with

$$
\Delta \tilde{T}=\frac{2 \Delta T}{h}, \quad \tilde{\gamma}_{l}=\frac{2 \gamma_{l}}{h}, \quad \tilde{\vartheta}_{a}=\frac{2 \vartheta_{a}}{h}, \quad \tilde{\nu}_{e}=\frac{2\left(d_{e} h-e \Delta t\right)}{h} .
$$

In each element, the SE method approximates solution segments $\mathbf{z}_{l}$ by their Lagrange interpolants as $\mathbf{z}_{l} \approx \tilde{\mathbf{z}}_{l}$, where

$$
\tilde{\mathbf{z}}_{l}=\sum_{k=1}^{N+1} \phi_{k}(\zeta) \tilde{\mathbf{z}}_{l, k},
$$

with $\tilde{\mathbf{z}}_{l, k}=\tilde{\mathbf{z}}_{l}\left(\zeta_{k}\right)$ and $\left\{\zeta_{k}\right\}_{k=1}^{N+1}$ being the set of interpolation grid points, which is chosen to be the Legendre-Gauss-Lobatto points. The system of operator equations (31) is multiplied with test functions $\left\{\psi_{i}(\zeta)\right\}_{i=1}^{N}$ and integrated over the domain $\zeta \in[-1,1]$ according to the standard steps of the weighted residual method. The test functions are chosen to be the Legendre polynomials up to degree $N-1$. With the above considerations equations (31)-(32) are discretized as

$$
\begin{aligned}
\sum_{k=1}^{N+1} \mathbf{S}_{i, k}^{l} \tilde{\mathbf{z}}_{l, k}=\sum_{a=1}^{m} \sum_{k=1}^{N+1} & \left(\mathbf{Q}_{i, k}^{l, a} \tilde{\mathbf{z}}_{l-r_{a}-1, k}+\mathbf{R}_{i, k}^{l, a} \tilde{\mathbf{z}}_{l-r_{a}, k}\right) \\
& +\sum_{b=0}^{n} \sum_{j=j_{l-1}}^{j_{l}} \sum_{k=1}^{N+1} \mathbf{P}_{i, k}^{l, j, b} \tilde{\mathbf{z}}_{d_{j v-b}, k}, \quad i=1,2, \ldots, N ; \quad l=1,2, \ldots, E \rho ;
\end{aligned}
$$

and

$$
\tilde{\mathbf{z}}_{l+1,1}=\tilde{\mathbf{z}}_{l, N+1}, \quad l=-\Gamma E \rho+1,-\Gamma E \rho+2, \ldots, E \rho-1 ;
$$

respectively, where

$$
\begin{aligned}
\mathbf{S}_{i, k}^{l} & =\int_{-1}^{1}\left(\frac{2}{h} \phi_{k}^{\prime}(\zeta) \mathbf{I}-\phi_{k}(\zeta) \mathbf{A}\left(\frac{h}{2}(\zeta+1)+h(l-1)\right)\right) \psi_{i}(\zeta) \mathrm{d} \zeta, \\
\mathbf{Q}_{i, k}^{l, a} & =\int_{-1}^{-1+\tilde{\vartheta}_{a}} \mathbf{B}_{a}\left(\frac{h}{2}(\zeta+1)+h(l-1)\right) \phi_{k}\left(\zeta+2-\tilde{\vartheta}_{a}\right) \psi_{i}(\zeta) \mathrm{d} \zeta, \\
\mathbf{R}_{i, k}^{l, a} & =\int_{-1+\tilde{\vartheta}_{a}}^{1} \mathbf{B}_{a}\left(\frac{h}{2}(\zeta+1)+h(l-1)\right) \phi_{k}\left(\zeta-\tilde{\vartheta}_{a}\right) \psi_{i}(\zeta) \mathrm{d} \zeta, \\
\mathbf{P}_{i, k}^{l, j, b} & =\mathbf{C}_{b} \phi_{k}\left(1-\tilde{\nu}_{j v-b}\right) \int_{\tilde{\kappa}_{1}^{l, j}}^{\tilde{\kappa}_{2}^{l, j}} \psi_{i}(\zeta) \mathrm{d} \zeta .
\end{aligned}
$$


Consequently, the numerical evaluation of integral terms in $40-(43)$ give

$$
\begin{aligned}
\mathbf{S}_{i, k}^{l} & =\mathbf{I} \frac{2}{h} \sum_{q=1}^{N+1} \phi_{k}^{\prime}\left(\eta_{q}\right) \psi_{i}\left(\eta_{q}\right) w_{q}-\mathbf{A}\left(\frac{h}{2}\left(\eta_{k}+1\right)+h(l-1)\right) \psi_{i}\left(\eta_{k}\right) w_{k}, \\
\mathbf{Q}_{i, k}^{l, a} & =\frac{\tilde{\vartheta}_{a}}{2} \sum_{q=1}^{N+1} \mathbf{B}_{a}\left(\frac{\vartheta_{a}}{2}\left(\eta_{q}+1\right)+h(l-1)\right) \phi_{k}\left(\frac{\tilde{\vartheta}_{a}}{2}\left(\eta_{q}-1\right)+1\right) \psi_{i}\left(\frac{\tilde{\vartheta}_{a}}{2}\left(\eta_{q}+1\right)-1\right) w_{q}, \\
\mathbf{R}_{i, k}^{l, a} & =\frac{2-\tilde{\vartheta}_{a}}{2} \sum_{q=1}^{N+1} \mathbf{B}_{a}\left(\frac{h-\vartheta_{a}}{2}\left(\eta_{q}+1\right)+\vartheta_{a}+h(l-1)\right) \phi_{k}\left(\frac{2-\tilde{\vartheta}_{a}}{2} \eta_{q}-\frac{\tilde{\vartheta}_{a}}{2}\right) \psi_{i}\left(\frac{2-\tilde{\vartheta}_{a}}{2} \eta_{q}+\frac{\tilde{\vartheta}_{a}}{2}\right) w_{q}, \\
\mathbf{P}_{i, k}^{l, j, b} & =\mathbf{C}_{b} \phi_{k}\left(1-\tilde{\nu}_{j v-b}\right) \frac{\tilde{\kappa}_{2}^{l, j}-\tilde{\kappa}_{1}^{l, j}}{2} \sum_{q=1}^{N+1} \psi_{i}\left(\frac{\tilde{\kappa}_{2}^{l, j}-\tilde{\kappa}_{1}^{l, j}}{2} \eta_{q}+\frac{\tilde{\kappa}_{2}^{l, j}+\tilde{\kappa}_{1}^{l, j}}{2}\right) w_{q},
\end{aligned}
$$

The non-zero parts of the matrix approximation $\mathbf{U} \in \mathbb{R}^{s(1+E \rho \Gamma N) \times s(1+E \rho \Gamma N)}$ of the monodromy operator are given by

$$
\begin{aligned}
& \mathbf{U}(1: s E \rho(\Gamma-1) N, s E \rho N+1: s E \rho \Gamma N)=\mathbf{I}, \\
& \mathbf{U}(s E \rho(\Gamma-1) N+1: s+s E \rho \Gamma N,:)=\boldsymbol{\Lambda}^{-1} \Upsilon \mathbf{},
\end{aligned}
$$

where the non-zero parts of matrix $\Lambda \in \mathbb{R}^{s(1+E \rho N) \times s(1+E \rho N)}$ are calculated according to

$$
\begin{aligned}
& \boldsymbol{\Lambda}(1: s, 1: s)=\mathbf{I}, \\
& \boldsymbol{\Lambda}(s+s(l-1) N+1: s+s l N, 1: s+s E \rho N)=\boldsymbol{\Lambda}_{l}, \quad l=1,2, \ldots, E \rho ;
\end{aligned}
$$

with

$$
\boldsymbol{\Lambda}_{l}=\boldsymbol{\Lambda}_{l}^{S}+\sum_{a=1}^{m}\left(\boldsymbol{\Lambda}_{l, a}^{Q}+\boldsymbol{\Lambda}_{l, a}^{R}\right)+\sum_{b=0}^{n} \sum_{j=j_{l-1}}^{j_{l}} \boldsymbol{\Lambda}_{l, j, b}^{P}
$$

and

$$
\begin{aligned}
& \Lambda_{l}^{S}(:, s(l-1) N+1: s+s l N)=\left[\mathbf{S}_{i, k}^{l}\right]_{i=1, k=1}^{N, N+1}, \\
& \boldsymbol{\Lambda}_{l, a}^{Q}\left(:, s\left(l-r_{a}-2\right) N+1: s+s\left(l-r_{a}-1\right) N\right)=\left[-\mathbf{Q}_{i, k}^{l, a}\right]_{i=1, k=1}^{N, N+1}, \text { if } l \geq r_{a}+2, \\
& \boldsymbol{\Lambda}_{l, a}^{R}\left(:, s\left(l-r_{a}-1\right) N+1: s+s\left(l-r_{a}\right) N\right)=\left[-\mathbf{R}_{i, k}^{l, a}\right]_{i=1, k=1}^{N, N+1}, \text { if } l \geq r_{a}+1, \\
& \Lambda_{l, j, b}^{P}\left(:, s\left(d_{j v-b}-1\right) N+1: s+s d_{j v-b} N\right)=\left[-\mathbf{P}_{i, k}^{l, j, b}\right]_{i=1, k=1}^{N, N+1}, \text { if } d_{j v-b} \geq 1,
\end{aligned}
$$

The first and second arguments of the above matrices indicate the indices of the rows and the columns, respectively, according to Matlab syntax. The non-zero parts of matrix $\Upsilon \in$ $\mathbb{R}^{s(1+N E \rho) \times s(1+N E \rho \Gamma)}$ are calculated according to

$$
\begin{aligned}
& \Upsilon(1: s, s E \rho \Gamma N+1: s+s E \rho \Gamma N)=\mathbf{I} \\
& \Upsilon(s+s(l-1) N+1: s+s l N, 1: s+s E \rho \Gamma N)=\Upsilon_{l}, \quad l=1,2, \ldots, E \rho
\end{aligned}
$$

where

$$
\mathbf{\Upsilon}_{l}=\sum_{a=1}^{m}\left(\mathbf{\Upsilon}_{l, a}^{Q}+\Upsilon_{l, a}^{R}\right)+\sum_{b=0}^{n} \sum_{j=j_{l-1}}^{j_{l}} \mathbf{\Upsilon}_{l, j, b}^{P}
$$

with

$$
\begin{aligned}
& \mathbf{\Upsilon}_{l, a}^{Q}\left(:, s\left(E \rho \Gamma+l-r_{a}-2\right) N+1: s+s\left(E \rho \Gamma+l-r_{a}-1\right) N\right)=\left[\mathbf{Q}_{i, k}^{l, a}\right]_{i=1, k=1}^{N, N+1}, \text { if } l<r_{a}+2, \\
& \mathbf{\Upsilon}_{l, a}^{R}\left(:, s\left(E \rho \Gamma+l-r_{a}-1\right) N+1: s+s\left(E \rho \Gamma+l-r_{a}\right) N\right)=\left[\mathbf{R}_{i, k}^{l, a}\right]_{i=1, k=1}^{N, N+1}, \text { if } l<r_{a}+1, \\
& \mathbf{\Upsilon}_{l, j, b}^{P}\left(:, s\left(E \rho \Gamma+d_{j v-b}-1\right) N+1: s+s\left(E \rho \Gamma+d_{j v-b}\right) N\right)=\left[\mathbf{P}_{i, k}^{l, j, b}\right]_{i=1, k=1}^{N, N+1}, \text { if } d_{j v-b}<1 .
\end{aligned}
$$


In summary, the steps of the computation of $\mathbf{U}$ are the following. First, the parameters in (21), (29) and (34)-(36) are calculated, then the terms in (44) are computed for all indices. Thereafter matrices $\boldsymbol{\Lambda}$ and $\boldsymbol{\Upsilon}$ are assembled according to (46)-(48) and (49)-(51), respectively. Finally, the matrix approximation $\mathbf{U}$ of the monodromy operator is constructed as (45).

In both the PT and the SE methods, the eigenvalues of matrix $\mathbf{U}$ approximate a finite number of characteristic multipliers of the monodromy operator $\mathcal{U}$. The eigenvalues of $\mathbf{U}$ can be calculated using the standard algorithms of numerical linear algebra. In the following section, stability diagrams are determined for engineering problems by checking the largest in-modulus eigenvalue of the matrix approximation $\mathbf{U}$ computed by the PT or the SE method. A point in the stability diagram is marked stable if the the largest in-modulus eigenvalue of $\mathbf{U}$ has a magnitude less than one. 


\section{Applications}

This section presents two engineering applications for the numerical methods detailed in Section 3. First, an extended mathematical model of a coupled haptic system is presented and stability analysis is performed. Thereafter, the mathematical model of the milling process subjected to active damping is investigated, where the sampling effect and zero-order hold of the control loop are taken into account.

\subsection{Coupled haptic system}

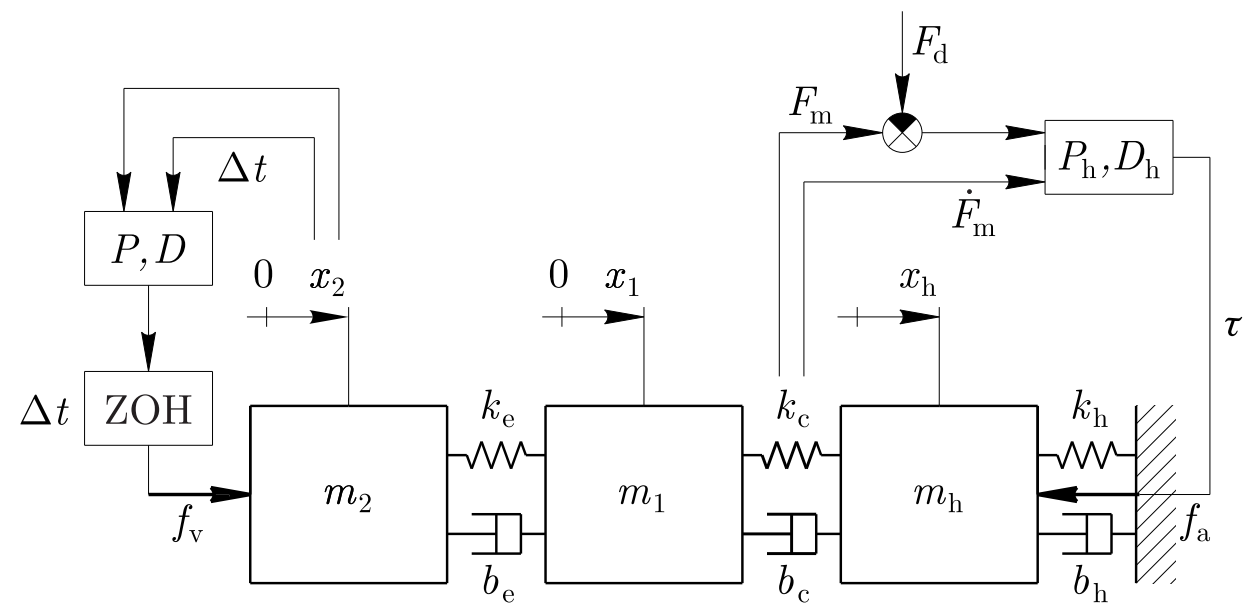

Figure 1: Concentrated parameter model of the haptic device based on [15] with simultaneous consideration of continuous delay $\tau$ in the human interaction and discrete delay $\Delta t$ in the digital controller.

As it was mentioned in Section 1, stability analysis has not yet been performed for haptic systems with the consideration of both continuous delay from human interaction and discrete delay from the control input. Based on the mechanical model presented in [15], this subsection derives a mathematical model, which takes into account both the delayed human interaction and the digital control. The formulas given in Section 3 are applied to the mathematical model and stability diagrams are computed in the space of control parameters.

Figure 1 shows the concentrated parameter model of the device in the rendered direction. Coordinates $x_{1}(t)$ and $x_{2}(t)$ describe the motion in the rendered subspace from the view point of the operator and from the view point of the virtual environment (actuator), respectively. The motion of the human hand in the rendered direction is given by $x_{\mathrm{h}}(t)$. The effective masses $m_{1}$ and $m_{2}$ characterize the inertia properties of the haptic device in the rendered subspace, while parameters $k_{\mathrm{e}}$ and $b_{\mathrm{e}}$ are the effective stiffness and damping values for the rendered direction. In the rendered subspace, the effective mass of the human hand is $m_{\mathrm{h}}$.

In [15], this concentrated parameter model was analyzed with a passive human interaction force. Here, an active human interaction force $f_{\mathrm{a}}$ is modeled, which is provided by a feedback force control mechanism. As shown in Figure 4 in [15], the model with a passive human interaction force cannot fully explain the increased stability observed in experiments. In [15], the authors proposed that the stabilizing effect of humans' active control can be modeled with a delayed proportional-differential (PD) control, which was demonstrated using a single mass-spring system with a natural frequency matching the dominant vibration frequency of the passive system at the loss of stability. Here, a unified model involving both the haptic mechanism of [15] and the active human control $f_{\mathrm{a}}$ is analyzed. 
The model shown in Figure 1 incorporates two human interaction force terms. The passive human interaction force

$$
f_{\mathrm{c}}(t)=k_{\mathrm{c}}\left(x_{1}(t)-x_{\mathrm{h}}(t)\right)+b_{\mathrm{c}}\left(\dot{x}_{1}(t)-\dot{x}_{\mathrm{h}}(t)\right)
$$

characterized by grasp stiffness $k_{\mathrm{c}}$ and damping $b_{\mathrm{c}}$, results from the visco-elastic contact between the human tissue and the endpoint link of the haptic device. In addition to the contact properties, the active human interaction force

$$
f_{\mathrm{a}}(t)=P_{\mathrm{h}}\left(F_{\mathrm{m}}(t-\tau)-F_{\mathrm{d}}\right)+D_{\mathrm{h}} \dot{F}_{\mathrm{m}}(t-\tau),
$$

aims to capture the feedback mechanism of the human behavior. This is equivalent to a force control model with a desired contact force $F_{\mathrm{d}}$, where the human operator measures the stiffnessrelated contact force component

$$
F_{\mathrm{m}}(t)=k_{\mathrm{c}}\left(x_{1}(t)-x_{\mathrm{h}}(t)\right)
$$

by tactile receptors. The human force control is modeled by a delayed PD control, where $P_{\mathrm{h}}$ and $D_{\mathrm{h}}$ are the proportional and derivative feedback gains, respectively, while $\tau$ captures the reaction delay between perception and muscle activation. Note that as [15] explains, during experiments humans were asked to keep a constant contact force with the haptic device, which motivated the employment of force feedback in $f_{\mathrm{a}}(t)$.

In addition to the active human interaction force, another control force is acting on the haptic device from the actuator side: the digital control force

$$
f_{\mathrm{v}}(t)=-P x_{2}\left(t_{j}\right)-D \frac{x_{2}\left(t_{j}\right)-x_{2}\left(t_{j}-\Delta t\right)}{\Delta t}, \quad t \in\left[t_{j}, t_{j}+\Delta t\right),
$$

which provides the virtual environment for the human operator. The control force is produced according to a PD feedback rule, where the derivative term is computed using the difference between two consecutive position samples. The proportional and derivative gains of the controller are denoted by $P$ and $D$, respectively.

Based on the concentrated parameter model shown in Figure 1, the governing equations are

$$
\begin{aligned}
& m_{1} \ddot{x}_{1}(t)+b_{\mathrm{e}}\left(\dot{x}_{1}(t)-\dot{x}_{2}(t)\right)+k_{\mathrm{e}}\left(x_{1}(t)-x_{2}(t)\right)=-f_{\mathrm{c}}(t), \\
& m_{2} \ddot{x}_{2}(t)+b_{\mathrm{e}}\left(\dot{x}_{2}(t)-\dot{x}_{1}(t)\right)+k_{\mathrm{e}}\left(x_{2}(t)-x_{1}(t)\right)=-\left(P+\frac{D}{\Delta t}\right) x_{2}\left(t_{j}\right)+\frac{D}{\Delta t} x_{2}\left(t_{j}-\Delta t\right), \\
& m_{\mathrm{h}} \ddot{x}_{\mathrm{h}}(t)=f_{\mathrm{c}}(t)-f_{\mathrm{a}}(t),
\end{aligned}
$$

where $t \in\left[t_{j}, t_{j}+\Delta t\right)$ and $f_{\mathrm{c}}(t)$ and $f_{\mathrm{a}}(t)$ are given in $(52)$ and $(53)$, respectively. In first-order form, the governing equations read as

$$
\dot{\mathbf{x}}(t)=\mathbf{A x}(t)+\mathbf{B} \mathbf{x}(t-\tau)+\mathbf{C}_{0} \mathbf{x}\left(t_{j}\right)+\mathbf{C}_{1} \mathbf{x}\left(t_{j}-\Delta t\right)+\mathbf{E}, \quad t \in\left[t_{j}, t_{j}+\Delta t\right),
$$


where the vectors and matrices are

$$
\begin{aligned}
& \mathbf{x}=\left[\begin{array}{llllll}
x_{1} & \dot{x}_{1} & x_{2} & \dot{x}_{2} & x_{\mathrm{h}} & \dot{x}_{\mathrm{h}}
\end{array}\right]^{\top}, \quad \mathbf{E}=\left[\begin{array}{llllll}
0 & 0 & 0 & 0 & 0 & -\frac{P_{\mathrm{h}} F_{\mathrm{d}}}{m_{\mathrm{h}}}
\end{array}\right]^{\top}, \\
& \mathbf{A}=\left[\begin{array}{cccccc}
0 & 1 & 0 & 0 & 0 & 0 \\
-\frac{k_{\mathrm{e}}+k_{\mathrm{c}}}{m_{1}} & -\frac{b_{\mathrm{e}}+b_{\mathrm{c}}}{m_{1}} & \frac{k_{\mathrm{e}}}{m_{1}} & \frac{b_{\mathrm{e}}}{m_{1}} & \frac{k_{\mathrm{c}}}{m_{1}} & \frac{b_{\mathrm{c}}}{m_{1}} \\
0 & 0 & 0 & 1 & 0 & 0 \\
\frac{k_{\mathrm{e}}}{m_{2}} & \frac{b_{\mathrm{e}}}{m_{2}} & -\frac{k_{\mathrm{e}}}{m_{2}} & -\frac{b_{\mathrm{e}}}{m_{2}} & 0 & 0 \\
0 & 0 & 0 & 0 & 0 & 1 \\
\frac{k_{\mathrm{c}}}{m_{\mathrm{h}}} & \frac{b_{\mathrm{c}}}{m_{\mathrm{h}}} & 0 & 0 & -\frac{k_{\mathrm{c}}}{m_{\mathrm{h}}} & -\frac{b_{\mathrm{c}}}{m_{\mathrm{h}}}
\end{array}\right] \\
& \mathbf{B}=\frac{k_{\mathrm{c}}}{m_{\mathrm{h}}}\left[\begin{array}{cccccc}
0 & 0 & 0 & 0 & 0 & 0 \\
0 & 0 & 0 & 0 & 0 & 0 \\
0 & 0 & 0 & 0 & 0 & 0 \\
0 & 0 & 0 & 0 & 0 & 0 \\
0 & 0 & 0 & 0 & 0 & 0 \\
P_{\mathrm{h}} & D_{\mathrm{h}} & 0 & 0 & -P_{\mathrm{h}} & -D_{\mathrm{h}}
\end{array}\right] \\
& \mathbf{C}_{0}=\left[\begin{array}{cccccc}
0 & 0 & 0 & 0 & 0 & 0 \\
0 & 0 & 0 & 0 & 0 & 0 \\
0 & 0 & 0 & 0 & 0 & 0 \\
0 & 0 & -\frac{P \Delta t+D}{m_{2} \Delta t} & 0 & 0 & 0 \\
0 & 0 & 0 & 0 & 0 & 0 \\
0 & 0 & 0 & 0 & 0 & 0
\end{array}\right], \quad \mathbf{C}_{1}=\left[\begin{array}{cccccc}
0 & 0 & 0 & 0 & 0 & 0 \\
0 & 0 & 0 & 0 & 0 & 0 \\
0 & 0 & 0 & 0 & 0 & 0 \\
0 & 0 & \frac{D}{m_{2} \Delta t} & 0 & 0 & 0 \\
0 & 0 & 0 & 0 & 0 & 0 \\
0 & 0 & 0 & 0 & 0 & 0
\end{array}\right]
\end{aligned}
$$

For the perturbation $\boldsymbol{\xi}(t)=\mathbf{x}(t)-\mathbf{x}_{\mathrm{e}}$ about equilibrium

$$
\mathbf{x}_{\mathrm{e}}=\left(\mathbf{A}+\mathbf{B}+\mathbf{C}_{0}+\mathbf{C}_{1}\right)^{-1} \mathbf{E},
$$

one obtains the hybrid DDE

$$
\dot{\boldsymbol{\xi}}(t)=\mathbf{A} \boldsymbol{\xi}(t)+\mathbf{B} \boldsymbol{\xi}(t-\tau)+\mathbf{C}_{0} \boldsymbol{\xi}\left(t_{j}\right)+\mathbf{C}_{1} \boldsymbol{\xi}\left(t_{j}-\Delta t\right), \quad t \in\left[t_{j}, t_{j}+\Delta t\right),
$$

which determines the stability of (57) about $\mathbf{x}_{\mathrm{e}}$.

Formulas derived in Section 3 can be applied to 60 for the computation of matrix approximation $\mathbf{U}$ of monodromy operator $\mathcal{U}$. The monodromy operator's largest-in-modulus eigenvalue, also called as dominant characteristic multiplier, uniquely determines the stability of (60) (for more details see Chapter 8 in [22]). This dominant multiplier is approximated by that of matrix $\mathbf{U}$. If the dominant multiplier of $\mathbf{U}$ converges with the increase of approximation parameters to that of $\mathcal{U}$, the stability of $(60)$ can be checked for fixed sets of system and control parameters by the computation of $\mathbf{U}$ at sufficiently high approximation parameters. Stability diagrams of (60) can thus be constructed over a grid of parameter plane $(P, D)$, where a grid point is marked stable if the dominant multiplier of $\mathbf{U}$ has absolute value less than one, otherwise the grid point is marked unstable. Using this method, first the convergence of stability boundaries is analyzed, then convergence of the dominant characteristic multiplier is checked at particular points of the parameter plane $(P, D)$.

Figures 24 show the stability boundaries of equation 60 computed according to Section 3 with parameters given in the corresponding captions of the figures and in Table 1. In Table 1 . $f_{\mathrm{s}}$ stands for the sampling frequency of the control loop of the haptic device, hence the sampling period is $\Delta t=1 / f_{\mathrm{s}}=0.00125[\mathrm{~s}]$.

Figures $2 \sqrt{3}$ show the stability boundaries computed by the PT and SE methods, respectively, with human control parameters $P_{\mathrm{h}}=0$ and $D_{\mathrm{h}}=0$, that is without active human interaction. If there is no active human interaction, then the term with continuous delay disappears in (60), 


\begin{tabular}{|c|l|}
\hline$f_{\mathrm{s}}$ & $800[\mathrm{~Hz}]$ \\
\hline$m_{1}$ & $0.2615[\mathrm{~kg}]$ \\
\hline$m_{2}$ & $0.0254[\mathrm{~kg}]$ \\
\hline$m_{\mathrm{h}}$ & $0.8[\mathrm{~kg}]$ \\
\hline$k_{\mathrm{e}}$ & $15620[\mathrm{~N} / \mathrm{m}]$ \\
\hline$k_{\mathrm{c}}$ & $1000[\mathrm{~N} / \mathrm{m}]$ \\
\hline$b_{\mathrm{e}}$ & $2[\mathrm{Ns} / \mathrm{m}]$ \\
\hline$b_{\mathrm{c}}$ & $1.6[\mathrm{Ns} / \mathrm{m}]$ \\
\hline
\end{tabular}

Table 1: System parameters used for the stability computations of (60)
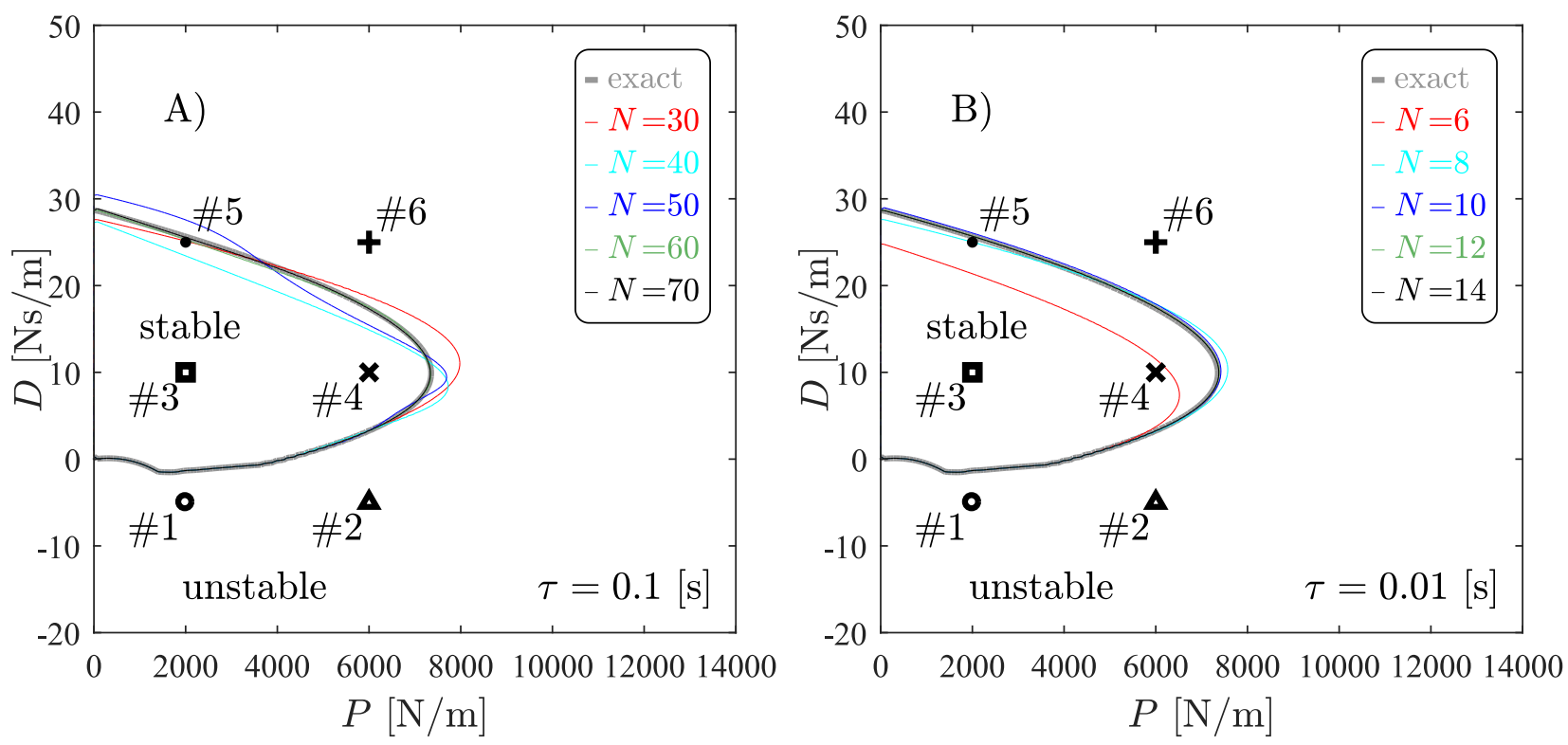

Figure 2: Stability diagrams, computed by the PT method, for the haptic system (60) without active human interaction $\left(P_{\mathrm{h}}=0, D_{\mathrm{h}}=0\right)$ and with parameters given according to Table 1. In panels A) and B), stability boundaries are shown for two different time history lengths $\tau$ with increasing order $N$ of polynomial approximation. The exact stability boundaries are shown with thick gray lines.

therefore the time length of history segment does not need to be equal to the human reaction delay $\tau$. In this case, both the exact monodromy operator $\mathcal{U}$ and the exact stability boundary can be calculated (see Figures 2 3) as detailed in [25].

The results in Figure 2 show that in case of the PT method, the closer the length $\tau$ of history segment is to its minimum length $\tau_{\min }=\Delta t$, the smaller order $N$ of polynomial approximation is required for accurate stability boundaries. Note that since $(60)$ incorporates no time-periodic coefficients, there is no need for the piecewise constant approximation of time-periodic terms in case of the PT method. As a result, $\tilde{v}=1$ is applied during stability computations. Due to the lack of time-periodic coefficients, the principal period $T_{\mathrm{p}}=\sigma \Delta t$ can be chosen as an arbitrary integer $\sigma \in \mathbb{Z}^{+}$multiple of the sampling period $\Delta t$. For the PT method $\sigma=1$ was used, while for the SE method $\sigma=10$ and $\sigma=20$ were applied.

Figure 3 shows the stability boundaries computed by the SE method for different $\sigma$ values, with changing order $N$ of polynomial approximation and with changing element number $E$. In contrast with the PT method, the convergence rate of stability boundaries, in case of the SE method, depends not on the length of time history segment but on the length of the principle 

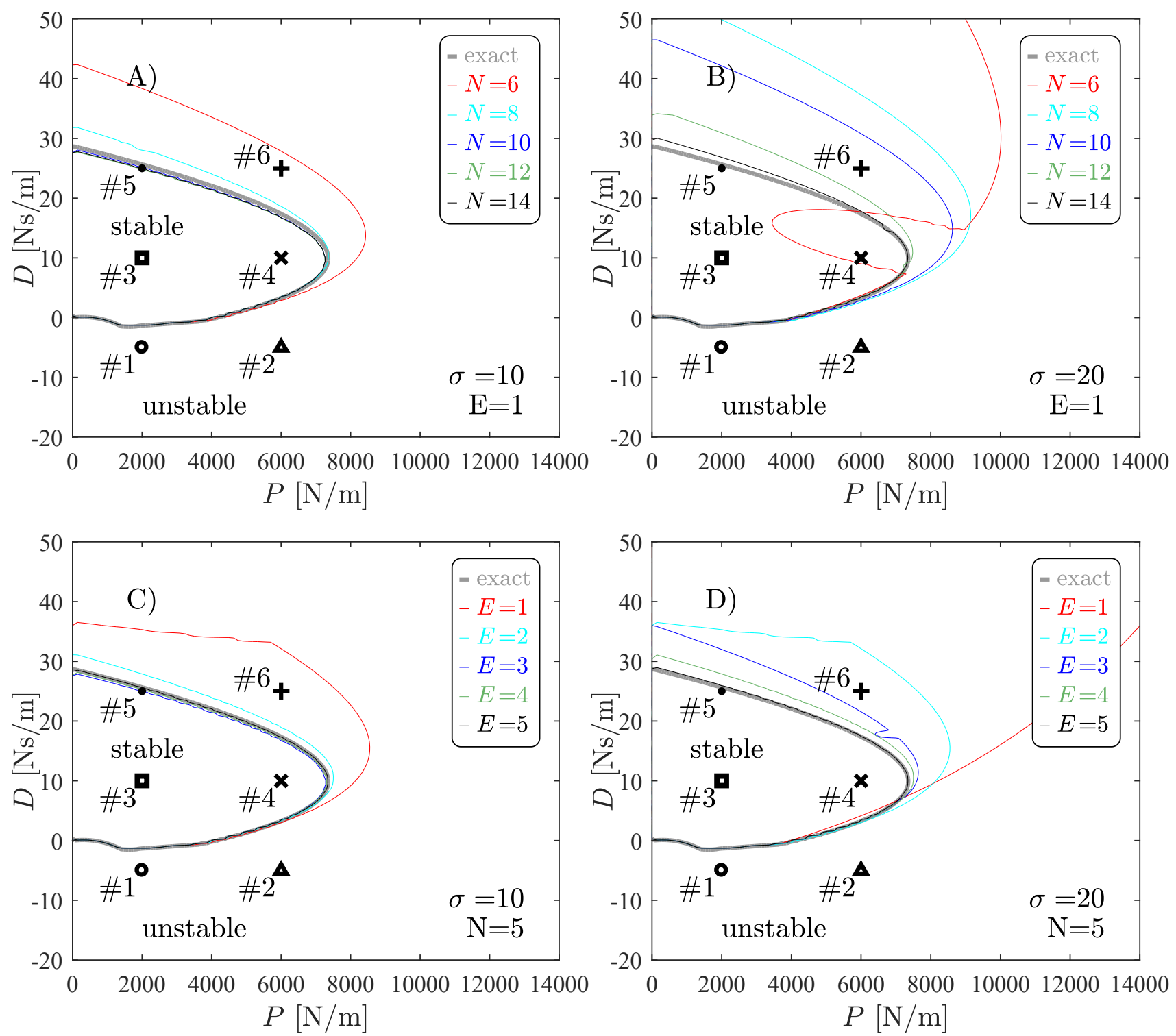

Figure 3: Stability diagrams, computed by the SE method, for the haptic system (60) without active human interaction $\left(P_{\mathrm{h}}=0, D_{\mathrm{h}}=0\right)$, with time history length $\tau=0.1[\mathrm{~s}]$ and system parameters given according to Table 1 . In panels A) and B), stability boundaries are shown with fixed element number $E$ and increasing order $N$ of polynomial approximation for two different principal periods $T_{\mathrm{p}}=\sigma \Delta t$. In panels $\mathrm{C}$ ) and $\mathrm{D}$ ), stability boundaries are shown with fixed order $N$ of polynomial approximation and increasing $E$ element number for two different principal periods. The exact stability boundaries are shown with thick gray lines.

period. As it can be inferred from Figure 3, longer principal period results slower convergence with respect to both the order $N$ of polynomial approximation and element number $E$.

Figure 4 shows the stability boundaries computed by the PT and SE methods with non-zero human control parameters, that is with active human interaction. It can be observed that with the increase of order $N$ of polynomial approximation, the stability boundaries converge to the same curve in case of both methods. As a reference, the case with no active human interaction is also plotted in Figure 4. It can be seen that the applied human control parameters affect the bottom part of the stability boundary, which corresponds to the experimental observations in 15.

Stability diagrams show only whether the system stays around its stationary state. However, it does not give insight into the decay of transients due to perturbations. In order to extract this 

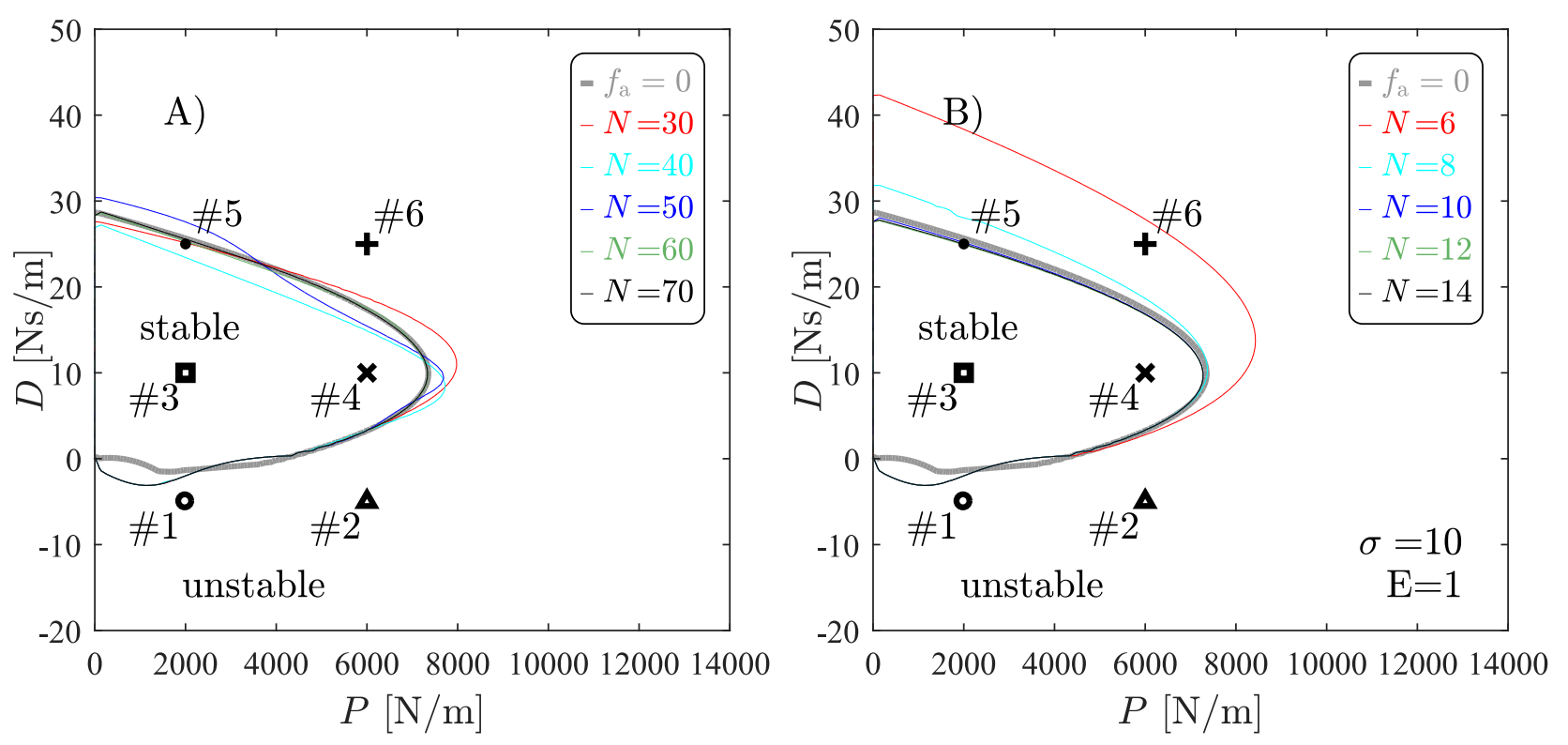

Figure 4: Stability diagrams, calculated by different numerical methods, for the haptic system (60) with active human interaction characterized by parameters $\tau=0.1[\mathrm{~s}], P_{\mathrm{h}}=-200[\mathrm{~N} / \mathrm{m}]$, $\overline{D_{\mathrm{h}}}=-5[\mathrm{Ns} / \mathrm{m}]$ and by parameters given according to Table 1. The stability boundaries in panels A) and B) were computed with the PT and SE methods, respectively, in both cases for increasing order $N$ of polynomial approximation. As reference, the exact stability boundary for the case of no active human interaction $\left(P_{\mathrm{h}}=0, D_{\mathrm{h}}=0\right)$ is shown with gray color.

information one should determine the absolute value of the dominant characteristic multiplier (for further details see [13]). In Figures 5 7, the normalized error

$$
e_{\mathrm{n}}(k)=\left|\frac{\mu_{k}-\mu^{*}}{\mu^{*}}\right|
$$

of dominant characteristic multipliers $\mu_{k}$ with respect to the accurate dominant characteristic multiplier $\mu^{*}$ is plotted in terms of approximation parameter $k$, in 6 points of the $(P, D)$ parameter plane. The coordinates of these points, marked in Figures 24 , are given in Table 2, where the absolute values of the accurate dominant multipliers employed for the construction of Figures 57 are shown as well. Note that only for the case without active human interaction force (that is when $P_{\mathrm{h}}=0$ and $D_{\mathrm{h}}=0$ ) can the exact dominant multiplier $\mu_{\text {exact }}^{*}$ be determined. Otherwise the dominant multipliers can only be approximated. Consequently, in the case of non-zero active human interaction force $\left(P_{\mathrm{h}}=-200[\mathrm{~N} / \mathrm{m}]\right.$ and $\left.D_{\mathrm{h}}=-5[\mathrm{Ns} / \mathrm{m}]\right)$ the accurate dominant multipliers were calculated using the two different numerical schemes of Section 3 at high approximation numbers. In particular, for the pseudospectral tau method, the accurate dominant multiplier $\mu_{\mathrm{PT}}^{*}$ was computed using $N=80$, while for the SE method, the accurate dominant multiplier $\mu_{\mathrm{SE}}^{*}$ was calculated using $N=80$ and $E=1$. As Table 2 and Figures 57 show, the absolute values of the dominant characteristic multipliers computed by the PT and SE methods converge to the same values in case of non-zero active human interaction force for all investigated points of control parameters with a normalized difference less than $0.1 \%$. Furthermore, both methods converge to the exact dominant multiplier $\mu_{\text {exact }}^{*}$ in case of no active human interaction.

In conclusion, it seems likely that the presented methods give results convergent to the exact stability boundaries and dominant characteristic multipliers of (60) in the case of active human interaction. This convergence property might hold even for sets of system parameters different from those in Table 1. However, in order to verify this conjecture one must perform a 


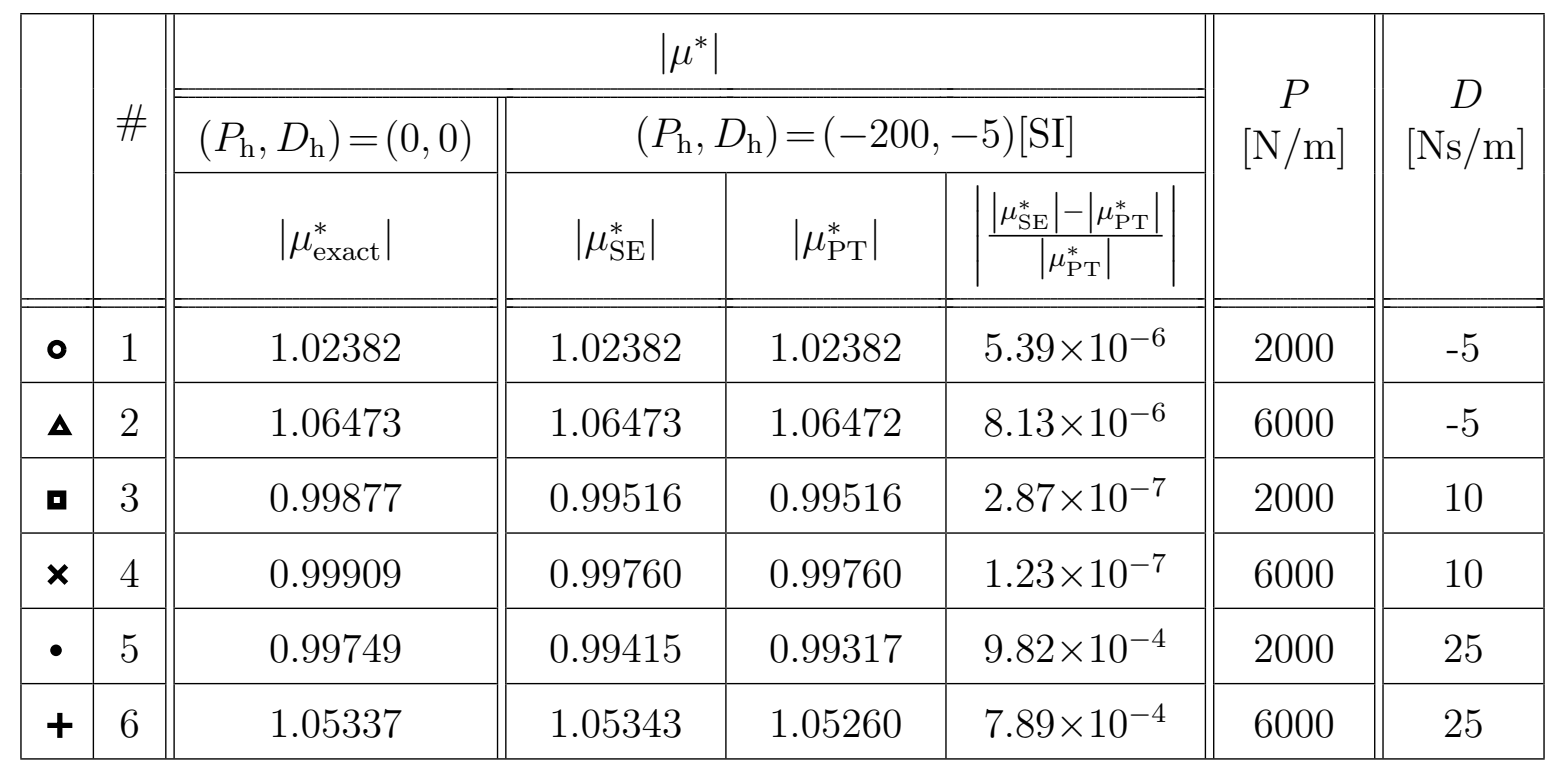

Table 2: Coordinates of the 6 investigated points of parameter plane $(P, D)$, marked in Figures 24. The accurate dominant characteristic multiplier $\mu^{*}$ is given by the exact dominant multiplier $\mu_{\text {exact }}^{*}$ for the case without active human interaction force (when $P_{\mathrm{h}}=0$ and $D_{\mathrm{h}}=0$ ) while it is given by the dominant characteristic multipliers $\mu_{\mathrm{SE}}^{*}$ and $\mu_{\mathrm{PT}}^{*}$ computed using the SE method with $N=80, E=1$ and the PT method with $N=80$, respectively, and assuming active human interaction force (when $P_{\mathrm{h}}=-200[\mathrm{~N} / \mathrm{m}]$ and $D_{\mathrm{h}}=-5[\mathrm{Ns} / \mathrm{m}]$ ).

precise theoretical convergence analysis, which is out of the scope of this paper. Nevertheless, the numerical results presented in this study can serve as a good base for a later theoretical convergence analysis.
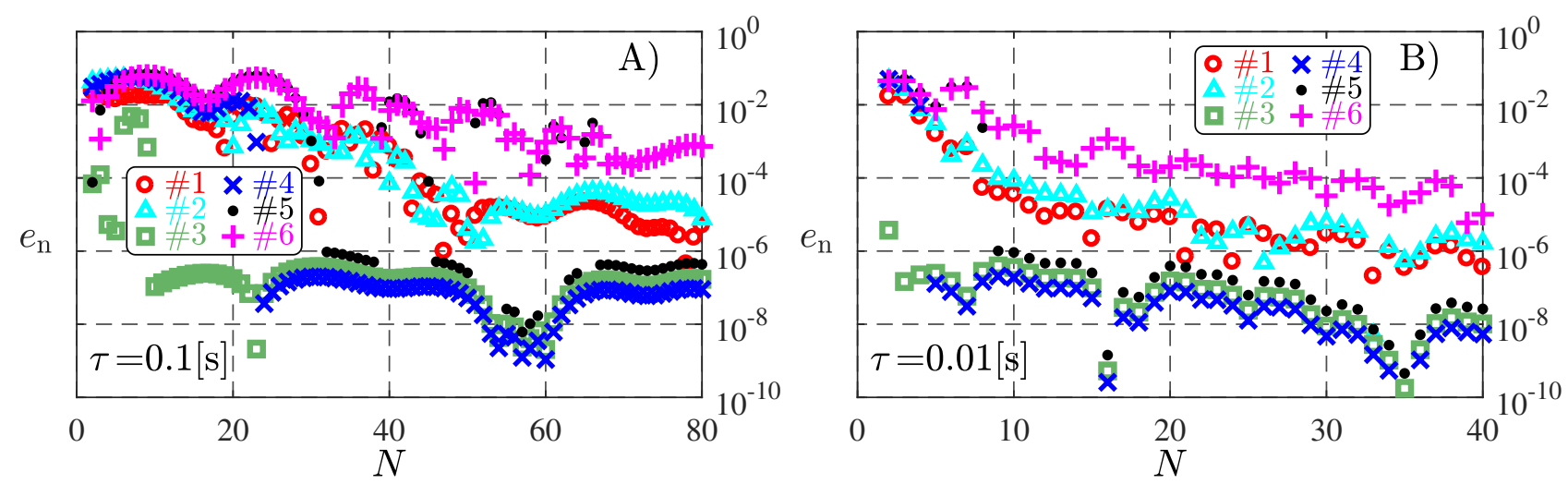

Figure 5: Convergence diagrams of the dominant characteristic multiplier corresponding to the respective panels of Figure 2. The diagrams show the normalized error $e_{\mathrm{n}}$ of the dominant characteristic multiplier in terms of polynomial order $N$ for 6 points of the $(P, D)$ parameter plane given in Table 2 and indicated in Figure 2, Diagrams were computed by the PT method for the case of no active human interaction $\left(P_{\mathrm{h}}=0, D_{\mathrm{h}}=0\right)$, using parameters according to Table 1 and $\tau=0.1[\mathrm{~s}]$ for panel A), and $\tau=0.01[\mathrm{~s}]$ for panel $\mathrm{B})$.

\subsection{Milling process with active damper}

In machining processes the large amplitude self-excited vibration between the workpiece and the tool is called machine tool chatter. One of the most accepted explanations for machine tool chatter is the so called regenerative effect [1, 26], which can be modeled by DDEs. So-called 

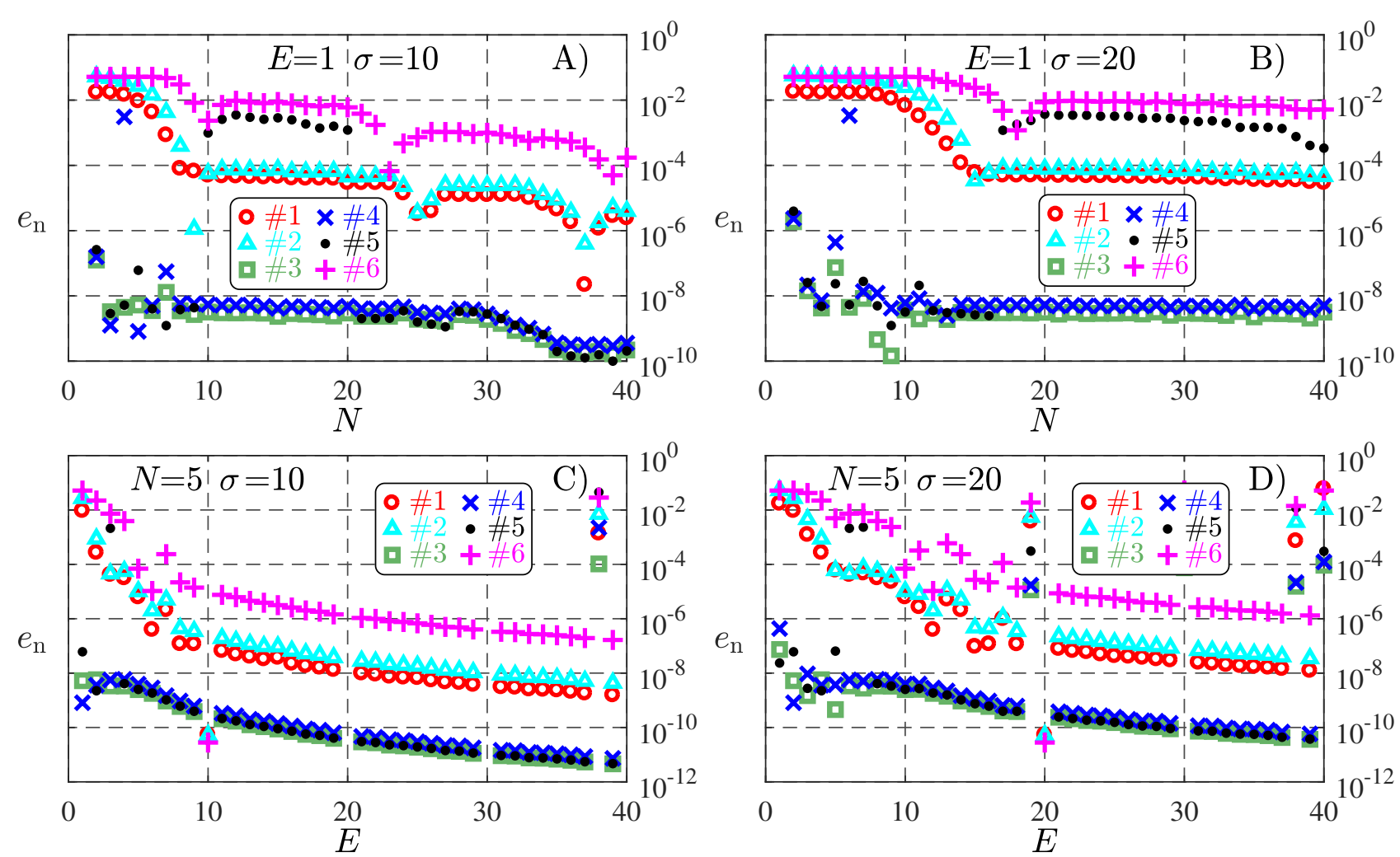

Figure 6: Convergence diagrams corresponding to the respective panels of Figure 3. The diagrams show the normalized error $e_{\mathrm{n}}$ of the dominant characteristic multiplier in terms of polynomial order $N$ and element number $E$ for 6 points of the $(P, D)$ parameter plane given in Table 2 and indicated in Figure 3. Diagrams were computed by the SE method for the case of no active human interaction $\left(P_{\mathrm{h}}=0, D_{\mathrm{h}}=0\right)$ using parameters given in Table 1 and $\tau=0.1$ [s]. In panels A) and C) $\sigma=10$, while in panels B) and D) $\sigma=20$ is applied.
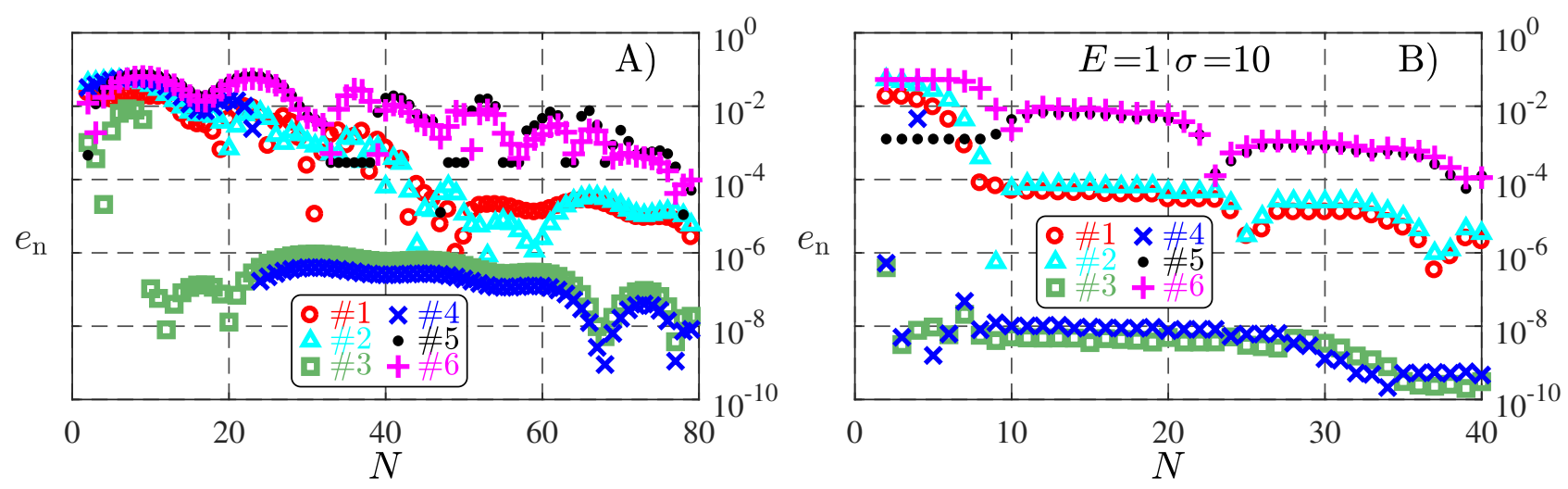

Figure 7: Convergence diagrams corresponding to the respective panels of Figure 4. Diagrams show the normalized error $e_{\mathrm{n}}$ of the dominant characteristic multiplier in terms of polynomial order $N$ for 6 points of the $(P, D)$ parameter plane given in Table 2 and indicated in Figure 4. Panels A) and B) were computed by the PT and SE methods, respectively, using parameters given in Table 1 and assuming active human interaction characterized by parameters $P_{\mathrm{h}}=-200$ $[\mathrm{N} / \mathrm{m}], D_{\mathrm{h}}=-5[\mathrm{Ns} / \mathrm{m}]$ and $\tau=0.1[\mathrm{~s}]$.

stability lobes diagrams (SLDs) are used to depict the regions associated with chatter-free machining in the parameter space of spindle speed $\Omega$ (in [rpm]) and axial depth of cut $a_{\mathrm{p}}$ (see Figure 8). In the machining literature it has been a subject of great interest how the 
stable domains in SLDs can be increased (thus chatter can be suppressed). There exist passive [27, 28], semi-active [29, 30] and active [31, 32] methods for the suppression of machine tool chatter. The active methods use a feedback loop, which usually involves a digital feedback controller in practice. However, apart from some studies presented for turning [33, 34], most existing models of active chatter suppression neglect the sampling effect and the zero-order hold of the feedback loop. Such study has not yet been presented for milling, mostly due to the required high computational effort of the stability analysis based on the existing standard methods of the literature. In this subsection, first a nonlinear mathematical model is derived for the milling process subjected to digital position control at the tool tip. Thereafter, stability analysis of the variational system around the stationary solution of this mathematical model is performed using the numerical methods detailed in Section 3 and results are visualized in the form of SLDs.
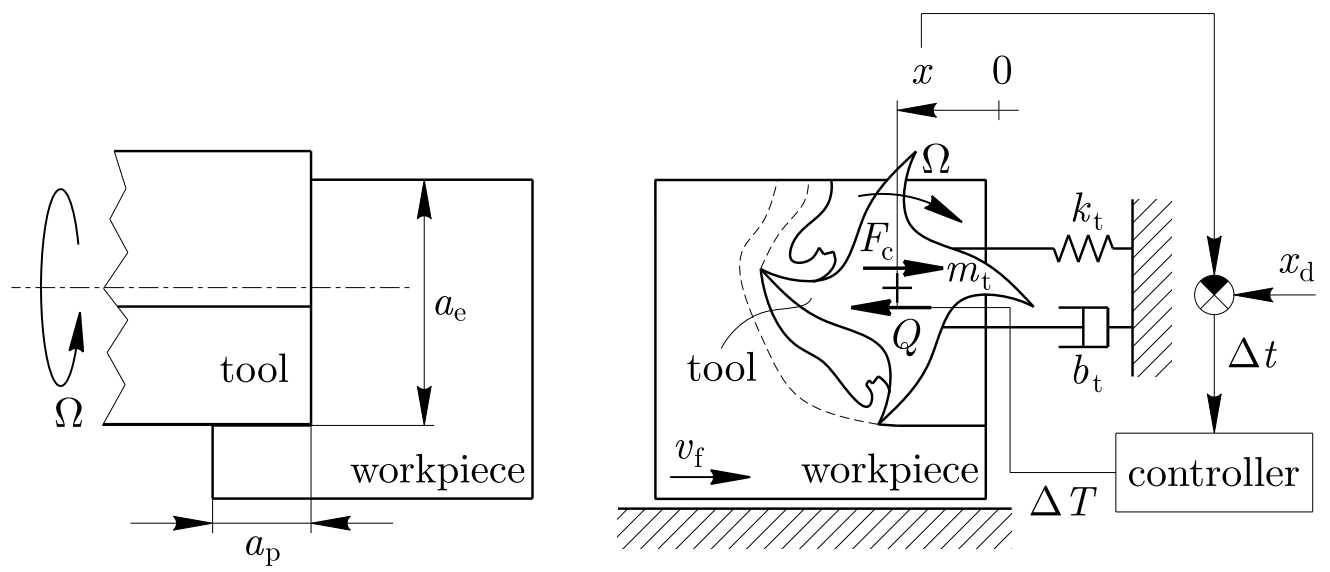

Figure 8: Model of milling subjected to active damping

One of the simplest models of regenerative vibrations in milling assumes that the tool (or the workpiece) can oscillate in the direction of the feed velocity only (for details see Chapter 5.2.1 in [8]). In addition, the model shown in Figure 8 takes into account a feedback loop controlled by a PD controller, which provides the active damping to the milling process. The displacement and the velocity of the tool tip are measured and used in the calculation of the control force $Q$ which acts at the tool tip. The tool is modeled by a block of mass $m_{\mathrm{t}}$, connected to the tool holder via a spring of stiffness $k_{\mathrm{t}}$ and a dash-pot of viscous damping $b_{\mathrm{t}}$ as shown in Figure 8. The workpiece is assumed to move horizontally with a constant feed velocity $v_{\mathrm{f}}$ relative to the tool holder. The undamped natural angular frequency of the tool is $\omega_{\mathrm{n}}=\sqrt{k_{\mathrm{t}} / m_{\mathrm{t}}}$ and the damping ratio is $\zeta=b_{\mathrm{t}} /\left(2 m_{\mathrm{t}} \omega_{\mathrm{n}}\right)$. Using dimensionless time $\bar{t}=\omega_{\mathrm{n}} t$ and dropping the bar immediately, the governing equations are

$$
\dot{\mathbf{x}}(t)=\mathbf{A}_{0} \mathbf{x}(t)+\mathbf{C}\left(\mathbf{x}\left(t_{j}-\delta t\right)-\mathbf{x}_{\mathrm{d}}\left(t_{j}-\delta t\right)\right)-\mathbf{f}\left(t, \mathbf{x}(t), \mathbf{x}\left(t-\tau_{\mathrm{d}}\right)\right), \quad t \in\left[t_{j}, t_{j}+\delta T\right),
$$

with $\delta t=\omega_{\mathrm{n}} \Delta t$ and $\delta T=\omega_{\mathrm{n}} \Delta T$ being the dimensionless sampling and actuation periods, respectively. The state $\mathbf{x}(t)$ and the desired trajectory $\mathbf{x}_{\mathrm{d}}(t)$ are defined as

$$
\mathbf{x}(t)=\left[\begin{array}{c}
x(t) \\
\dot{x}(t)
\end{array}\right], \quad \mathbf{x}_{\mathrm{d}}(t)=\left[\begin{array}{c}
x_{\mathrm{d}}(t) \\
\dot{x}_{\mathrm{d}}(t)
\end{array}\right],
$$


while

$$
\mathbf{A}_{0}=\left[\begin{array}{cc}
0 & 1 \\
-1 & -2 \zeta
\end{array}\right], \mathbf{C}=\left[\begin{array}{cc}
0 & 0 \\
-k_{P} & -k_{D}
\end{array}\right], \mathbf{f}\left(t, \mathbf{x}(t), \mathbf{x}\left(t-\tau_{\mathrm{d}}\right)\right)=\frac{F_{\mathrm{c}}\left(t, \mathbf{x}(t), \mathbf{x}\left(t-\tau_{\mathrm{d}}\right)\right)}{m_{\mathrm{t}} \omega_{\mathrm{n}}^{2}}\left[\begin{array}{l}
0 \\
1
\end{array}\right] .
$$

The controller uses PD control in order to stabilize the oscillator about the desired trajectory $\mathbf{x}_{\mathrm{d}}(t)$. The control force $Q$ consists of proportional and differential terms with feedback gains $P$ and $D$, respectively. Note that the difference between the measured and the desired trajectory is fed back with a delay $\delta t$ in the control force term. This delay accounts for the processing time of the measured data and for the estimation of the desired state. Due to the rescaled time, dimensionless control gains are introduced as $k_{P}=P /\left(m_{\mathrm{t}} \omega_{\mathrm{n}}^{2}\right)$ and $k_{D}=D /\left(m_{\mathrm{t}} \omega_{\mathrm{n}}\right)$. The dimensionless regenerative delay (which coincides with the tooth passing period) is $\tau_{\mathrm{d}}=2 \pi / \Omega_{\mathrm{d}}$, with $\Omega_{\mathrm{d}}=2 \pi \Omega Z /\left(60 \omega_{\mathrm{n}}\right)$ being the dimensionless spindle speed and $Z$ being the number of cutting teeth. The cutting force is the resultant of forces acting on the teeth (see Figure 9), hence the horizontal component of the resultant cutting force is

$$
\begin{aligned}
& F_{\mathrm{c}}\left(t, \mathbf{x}(t), \mathbf{x}\left(t-\tau_{\mathrm{d}}\right)\right)= \\
& \sum_{p=1}^{Z} g_{p}(t)\left(F_{\mathrm{t}, p}\left(t, \mathbf{x}(t), \mathbf{x}\left(t-\tau_{\mathrm{d}}\right)\right) \cos \left(\varphi_{p}(t)\right)+F_{\mathrm{n}, p}\left(t, \mathbf{x}(t), \mathbf{x}\left(t-\tau_{\mathrm{d}}\right)\right) \sin \left(\varphi_{p}(t)\right)\right),
\end{aligned}
$$

where the window function

$$
g_{p}(t)= \begin{cases}1 & \text { if } \varphi_{\mathrm{ent}} \leq \varphi_{p}(t) \bmod 2 \pi \leq \varphi_{\mathrm{ex}} \\ 0 & \text { otherwise }\end{cases}
$$

models whether the $p^{\text {th }}$ tooth is in or out of cut while $\varphi_{\text {ent }}$ and $\varphi_{\text {ex }}$ stand for the entrance angle and the exit angle of the cutting teeth. For up-milling operation $\varphi_{\text {ent }}=0$ and $\varphi_{\text {ex }}=$ $\arccos \left(1-2 a_{\mathrm{e}} / d\right)$, while for down-milling operation $\varphi_{\mathrm{ent}}=\arccos \left(2 a_{\mathrm{e}} / d-1\right)$ and $\varphi_{\mathrm{ex}}=\pi$, with $a_{\mathrm{e}}$ being the radial immersion (see Figure 8) and $d$ is the diameter of the tool (see Figure 9). The tangential and normal force components of the $p^{\text {th }}$ tooth are both calculated according to

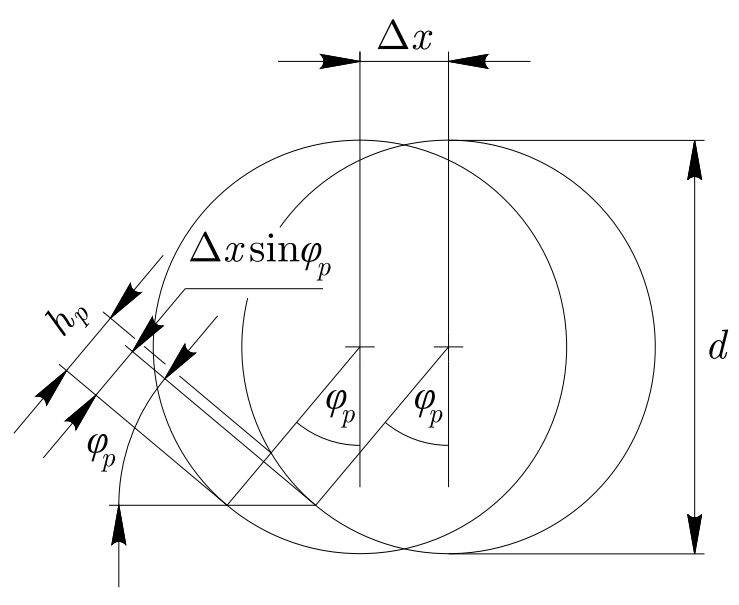

A)

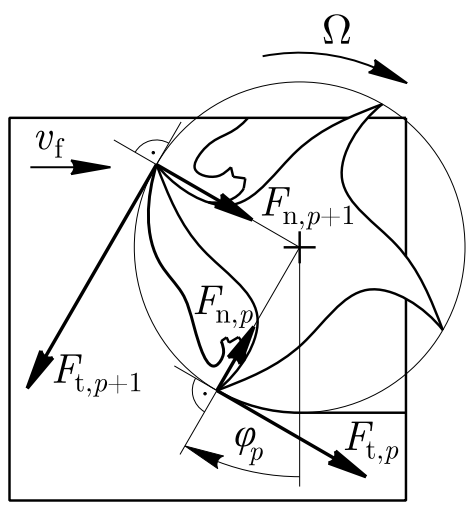

B)

Figure 9: Cutting model: A) circular tooth path approximation B) tangential and normal cutting force components 
Taylor's $3 / 4$ rule (see [35]) as

$$
\begin{aligned}
& F_{\mathrm{t}, p}\left(t, \mathbf{x}(t), \mathbf{x}\left(t-\tau_{\mathrm{d}}\right)\right)=a_{\mathrm{p}} K_{\mathrm{t}} h_{p}^{3 / 4}\left(t, \mathbf{x}(t), \mathbf{x}\left(t-\tau_{\mathrm{d}}\right)\right) \\
& F_{\mathrm{n}, p}\left(t, \mathbf{x}(t), \mathbf{x}\left(t-\tau_{\mathrm{d}}\right)\right)=a_{\mathrm{p}} K_{\mathrm{n}} h_{p}^{3 / 4}\left(t, \mathbf{x}(t), \mathbf{x}\left(t-\tau_{\mathrm{d}}\right)\right),
\end{aligned}
$$

respectively. Here the angular position of the $p^{\text {th }}$ tooth at dimensionless time instant $t$ is $\varphi_{p}(t)=\left(\Omega_{\mathrm{d}} t+(p-1) 2 \pi\right) / Z$, while the tangential and normal cutting force coefficients are $K_{\mathrm{t}}$ and $K_{\mathrm{n}}$, respectively. By assuming circular tooth path (see Figure 9), the chip thickness on the $p^{\text {th }}$ tooth at dimensionless time instant $t$ can be approximated as

$$
h_{p}\left(t, \mathbf{x}(t), \mathbf{x}\left(t-\tau_{\mathrm{d}}\right)\right) \approx \Delta x\left(\mathbf{x}(t), \mathbf{x}\left(t-\tau_{\mathrm{d}}\right)\right) \sin \left(\varphi_{p}(t)\right),
$$

where

$$
\Delta x\left(\mathbf{x}(t), \mathbf{x}\left(t-\tau_{\mathrm{d}}\right)\right)=f_{\mathrm{Z}}+x(t)-x\left(t-\tau_{\mathrm{d}}\right)
$$

is the difference in the relative position of the tool and the workpiece between two consecutive cuts while the feed per tooth rate is given by $f_{\mathrm{Z}}=\bar{v}_{\mathrm{f}} \tau_{\mathrm{d}}$, with $\bar{v}_{\mathrm{f}}=v_{\mathrm{f}} / \omega_{\mathrm{n}}$ being the specific feed velocity.

It is assumed that the stationary solution $\mathbf{x}_{\mathbf{s}}(t)$ for 62 is equal to the desired solution $\mathbf{x}_{\mathrm{d}}(t)$, which gives

$$
\dot{\mathbf{x}}_{\mathrm{s}}(t)=\mathbf{A}_{0} \mathbf{x}_{\mathrm{s}}(t)-\mathbf{f}\left(t, \mathbf{x}_{\mathrm{s}}(t), \mathbf{x}_{\mathrm{s}}\left(t-\tau_{\mathrm{d}}\right)\right) .
$$

Since $\mathbf{f}\left(t+\tau_{\mathrm{d}}, \cdot, \cdot\right)=\mathbf{f}(t, \cdot, \cdot)$, there exists a $\tau_{\mathrm{d}}$-periodic stationary solution $\mathbf{x}_{\mathrm{p}}(t)=\mathbf{x}_{\mathrm{p}}\left(t+\tau_{\mathrm{d}}\right)$ of (70). If the desired solution is set to be the periodic stationary solution of (70), that is $\mathbf{x}_{\mathrm{d}}(t)=\mathbf{x}_{\mathrm{p}}(t)$, then $\mathbf{f}\left(t, \mathbf{x}_{\mathrm{p}}(t), \mathbf{x}_{\mathrm{p}}\left(t-\tau_{\mathrm{d}}\right)\right)=\mathbf{f}_{\mathrm{p}}(t)$ becomes solely time-dependent (see (64)-(69) $)$. Consequently, (70) becomes a linear time-periodic ordinary differential equation for which the $\tau_{\mathrm{d}}$-periodic stationary solution $\mathbf{x}_{\mathrm{p}}(t)$ can be computed on a simple way. Here, it is important to note that in practice $\mathbf{x}_{\mathrm{p}}(t)$ and, consequently, $\mathbf{x}_{\mathbf{s}}(t)$ cannot be determined accurately due to modeling and parameter uncertainties, therefore the desired solution of the controller cannot be set equal to the stationary solution $\mathbf{x}_{\mathbf{S}}(t)$. This implies that the actual stationary solution is different from $\mathbf{x}_{\mathbf{s}}(t)$ and is determined by the nonlinear hybrid DDE (62). Nevertheless, here it is assumed that $\mathbf{x}_{\mathrm{d}}(t)=\mathbf{x}_{\mathrm{p}}(t)$ is known exactly as the $\tau_{\mathrm{d}}$-periodic stationary solution of (70). Consequently, linear stability properties can be analyzed by the variational system of 62) about the stationary solution $\mathbf{x}_{\mathrm{p}}(t)$.

In (62), the decomposition of state variables as $\mathbf{x}(t)=\mathbf{x}_{\mathbf{p}}(t)+\boldsymbol{\xi}(t)$ and the first-order Taylor expansion of $\mathbf{f}\left(t, \mathbf{x}(t), \mathbf{x}\left(t-\tau_{\mathrm{d}}\right)\right)$ about $\mathbf{x}_{\mathrm{p}}(t)$ with respect to perturbation $\boldsymbol{\xi}(t)$ leads to the variational system

$$
\dot{\boldsymbol{\xi}}(t)=\left(\mathbf{A}_{0}-\mathbf{B}(t)\right) \boldsymbol{\xi}(t)+\mathbf{B}(t) \boldsymbol{\xi}\left(t-\tau_{\mathrm{d}}\right)+\mathbf{C} \boldsymbol{\xi}\left(t_{j}-\delta t\right) \quad t \in\left[t_{j}, t_{j}+\delta T\right),
$$

where

$$
\mathbf{B}(t)=\left[\begin{array}{cc}
0 & 0 \\
H_{\mathrm{d}} & 0
\end{array}\right] \sum_{p=1}^{Z} B_{p}(t)
$$

and

$$
B_{p}(t)=g_{p}(t) \sin ^{3 / 4}\left(\varphi_{p}(t)\right)\left(K_{\mathrm{r}} \cos \left(\varphi_{p}(t)\right)+\sin \left(\varphi_{p}(t)\right)\right),
$$

with $H_{\mathrm{d}}=3 a_{\mathrm{p}} K_{\mathrm{n}} f_{\mathrm{Z}}^{-1 / 4} /\left(4 m_{\mathrm{t}} \omega_{\mathrm{n}}^{2}\right)$ being the dimensionless specific cutting force coefficient and $K_{\mathrm{r}}=K_{\mathrm{t}} / K_{\mathrm{n}}$ is the cutting force coefficient ratio.

Similarly as in Section 4.1, the stability of variational system (71) can be analyzed using the matrix approximation $\mathbf{U}$ of the monodromy operator $\mathcal{U}$ of (71). Matrix $\mathbf{U}$ can be constructed using the numerical methods presented in Section 3 and by the computation of its largestin-modulus eigenvalue the dominant characteristic multiplier of $\mathcal{U}$ can be approximated. In 


\begin{tabular}{|c|l|}
\hline$\zeta$ & 0.05 \\
\hline$K_{\mathrm{r}}$ & 3 \\
\hline$a_{\mathrm{e}} / D$ & 0.5 \\
\hline$Z$ & 2 \\
\hline
\end{tabular}

Table 3: System parameters used for the stability computations of (71)

\begin{tabular}{|c|c||c|c|c||c|c|c||c|}
\hline & \multirow{2}{*}{$\#$} & \multicolumn{3}{|c|}{$\left|\mu^{*}\right|$} & $\rho$ & $\sigma$ & $\Omega_{\mathrm{d}}=\frac{2 \pi \rho}{\delta t \sigma}$ & $H_{\mathrm{d}}$ \\
\cline { 3 - 7 } & & $\left|\mu_{\mathrm{SE}}^{*}\right|$ & $\left|\mu_{\mathrm{PT}}^{*}\right|$ & $\mid \frac{\left|\mu_{\mathrm{SE}}^{*}\right|-\left|\mu_{\mathrm{PT}}^{*}\right|}{\left|\mu_{\mathrm{PT}}^{*}\right|}$ & & & & \\
\hline \hline $\mathbf{0}$ & 1 & 0.90572 & 0.90436 & $1.50 \times 10^{-3}$ & 74 & 5 & 0.8491 & 0.3 \\
\hline $\mathbf{\Delta}$ & 2 & 0.59035 & 0.59031 & $6.82 \times 10^{-5}$ & 9 & 1 & 1.3963 & 0.3 \\
\hline $\mathbf{\square}$ & 3 & 0.60778 & 0.60732 & $7.66 \times 10^{-4}$ & 58 & 9 & 1.9500 & 0.3 \\
\hline $\mathbf{x}$ & 4 & 8.40669 & 8.39782 & $1.06 \times 10^{-3}$ & 74 & 5 & 0.8491 & 0.75 \\
\hline - & 5 & 0.83694 & 0.83708 & $1.60 \times 10^{-4}$ & 9 & 1 & 1.3963 & 0.75 \\
\hline $\mathbf{+}$ & 6 & 41.99581 & 41.97366 & $5.28 \times 10^{-4}$ & 58 & 9 & 1.9500 & 0.75 \\
\hline
\end{tabular}

Table 4: Coordinates of the 6 investigated points of parameter plane $\left(\Omega_{\mathrm{d}}, H_{\mathrm{d}}\right)$, marked in Figure 10. The accurate dominant characteristic multiplier $\mu^{*}$ is given by the dominant characteristic multipliers $\mu_{\mathrm{SE}}^{*}$ and $\mu_{\mathrm{PT}}^{*}$ computed using the SE method with $N=80, E=1$ and the PT method with $N=80, \tilde{v}=40$, respectively.

the following, first the convergence of stability boundaries is analyzed in the plane $\left(\Omega_{\mathrm{d}}, H_{\mathrm{d}}\right)$ of system parameters with respect to increasing approximation parameters. Thereafter, the convergence of the dominant characteristic multiplier is studied in 6 points of the parameter plane $\left(\Omega_{\mathrm{d}}, H_{\mathrm{d}}\right)$.

It is important to note that $\mathbf{x}_{\mathrm{p}}(t)$ is not involved in the variational system (71), therefore the periodic solution of 70 does not need to be determined for the construction of $\mathbf{U}$. It is also worth noting that in general $\mathbf{B}(t)$ is discontinuous with respect to time, which can destroy the convergence properties of the standard SE method. In this paper the problem given by the discontinuities in $\mathbf{B}(t)$ is solved by separating the integral terms in (41)-(42) at the discontinuity points of $B_{p}(t)$ (for details see [36]).

The period of functions $g_{p}(t)$ is $\tau_{\mathrm{d}}$ and the digital control introduces an additional time period: the dimensionless actuation period $\delta T=v \delta t, v \in \mathbb{Z}^{+}$. Consequently, (71) is a quasi-periodic system. Here, it is assumed that the ratio of the actuation period and the tooth-passing period is rational and a principal period can be given as $T_{\mathrm{p}}=\delta T \sigma=\tau_{\mathrm{d}} \rho$, with $\sigma, \rho \in \mathbb{Z}^{+}$. Thus, the case given in Section 2 can be applied.

The SLD approximations corresponding to (71) are shown in Figures 10 for a fixed parameter set given in the caption and in Table 3. Note that $v=1$ is assumed for all cases, which results that the actuation period is equal to the sampling period, that is $\delta T=\delta t$.

In panels A) and B) of Figure 10, the stability diagrams were computed using the PT method. There are two approximation parameters in the PT method: order $N$ of polynomial approximation and resolution $\tilde{v}$ of the dimensionless actuation period $\delta T$. The stability boundary converges if both $N$ and $\tilde{v}$ are increased, therefore sufficiently high $N$ and $\tilde{v}$ are required for an accurate stability boundary. In panels C) and D) of Figure 10, the SLDs were computed using the SE method. There are two approximation parameters in the SE method: order $N$ 

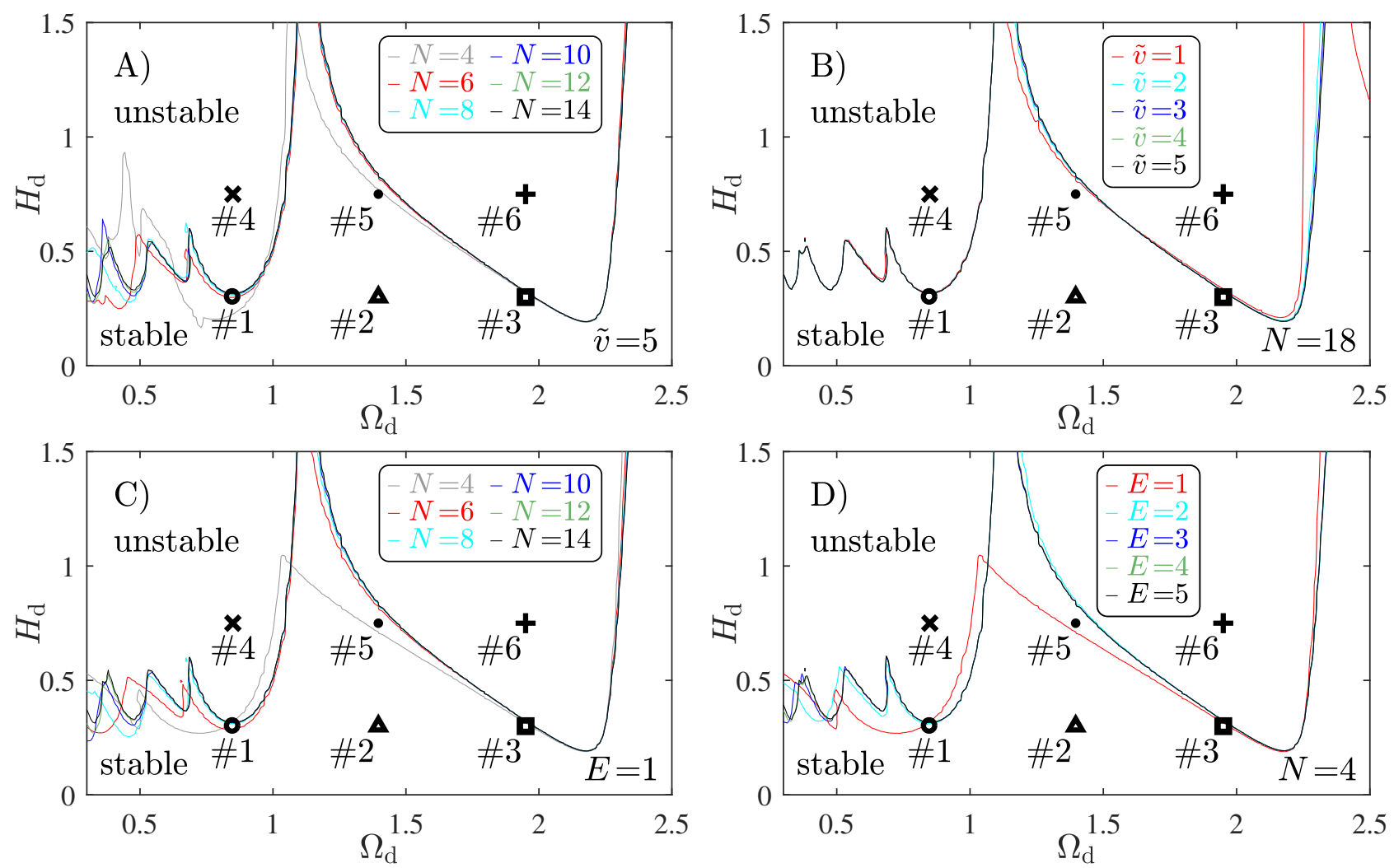

Figure 10: Stability lobes diagrams for down-milling operations with active damper using system parameters given in Table 3 and control parameters $v=1, k_{P}=0.2, k_{D}=0.2$, $\delta t=0.5$. In panels A) and B) stability boundaries are shown for the PT method with fixed resolution $\tilde{v}$ and order $N$ of polynomial approximation, respectively. In panels $\mathrm{C}$ ) and $\mathrm{D}$ ) stability boundaries are shown for the SE method with fixed element number $E$ and order $N$ of polynomial approximation, respectively.

of polynomial approximation and element number $E$, which is the number of elements within one dimensionless tooth passing period $\tau_{\mathrm{d}}$. The stability boundary converges if either $N$ or $E$ is increased, therefore sufficiently high $N$ or $E$ are required for an accurate stability boundary. Note that the difficulty of the SLD calculation is given by the change in the tooth passing period $\tau_{\mathrm{d}}=2 \pi / \Omega_{\mathrm{d}}$ along the axis $\Omega_{\mathrm{d}}$. Since dimensionless actuation period $\delta T$ is fixed, this results in different $T_{\mathrm{p}}=\delta T \sigma=\tau_{\mathrm{d}} \rho$ principal periods for each point along the axis $\Omega_{\mathrm{d}}$. Therefore the distribution of grid points along $\Omega_{\mathrm{d}}$ is not uniform, because $\sigma, \rho \in \mathbb{Z}^{+}$has to hold while the dimensionless spindle speed is computed as $\Omega_{\mathrm{d}}=2 \pi \rho /(\delta T \sigma)$.

Figure 12 shows the convergence of stability boundaries for fixed order $N$ of polynomial approximation and increasing $\tilde{v}$ of actuation period resolution. It can be inferred from panel B) in Figure 10 and from Figure 12 , that the higher the ratio $\delta t / \tau_{\mathrm{d}}$ of sampling period and time period of periodic coefficients, the higher resolution $\tilde{v}$ is necessary for sufficiently converged stability boundaries.

Similarly as in Section 4.1, in addition to the convergence of stability boundaries, the convergence of dominant multipliers is also investigated. In Figure 11, the normalized error (61) of dominant multipliers $\mu_{k}$ with respect to the accurate dominant multiplier $\mu^{*}$ is plotted in terms of approximation parameter $k$ in 6 points of the $\left(\Omega_{\mathrm{d}}, H_{\mathrm{d}}\right)$ parameter plane. The coordinates of these points, marked in Figure 10, are given in Table 4, where the absolute values of the accurate dominant multipliers employed for the construction of Figure 11 is shown as well. Due to that no exact solution can be determined for the monodromy operator and its dominant characteristic multiplier, the accurate dominant multipliers were computed using the 

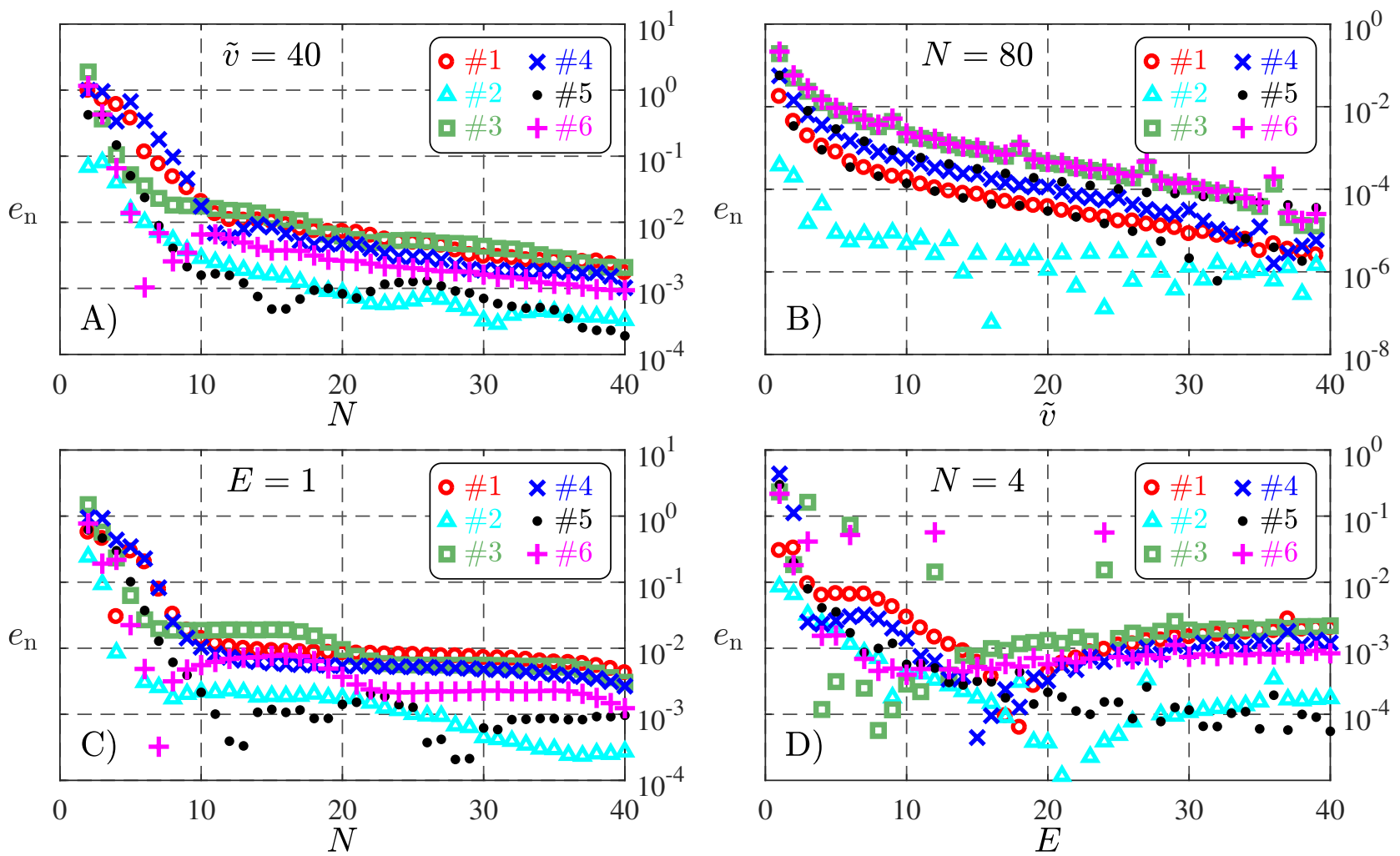

Figure 11: Convergence diagrams corresponding to the respective panels of Figure 10. Diagrams show the normalized error $e_{\mathrm{n}}$ of the dominant characteristic multiplier for system parameters given in Table 3 and control parameters $v=1, k_{P}=0.2, k_{D}=0.2, \delta t=0.5$. In panels A) and B) the normalized error is visualized for the PT method in terms of polynomial order $N$ and actuation period resolution $\tilde{v}$, respectively. In panels $\mathrm{C}$ ) and $\mathrm{D}$ ) the normalized error is shown for the SE method in terms of polynomial order $N$ and element number $E$, respectively. In each diagram, the normalized error is visualized for 6 points of the parameter plane $\left(\Omega_{\mathrm{d}}, H_{\mathrm{d}}\right)$, given in Table 4 and marked in Figure 10.
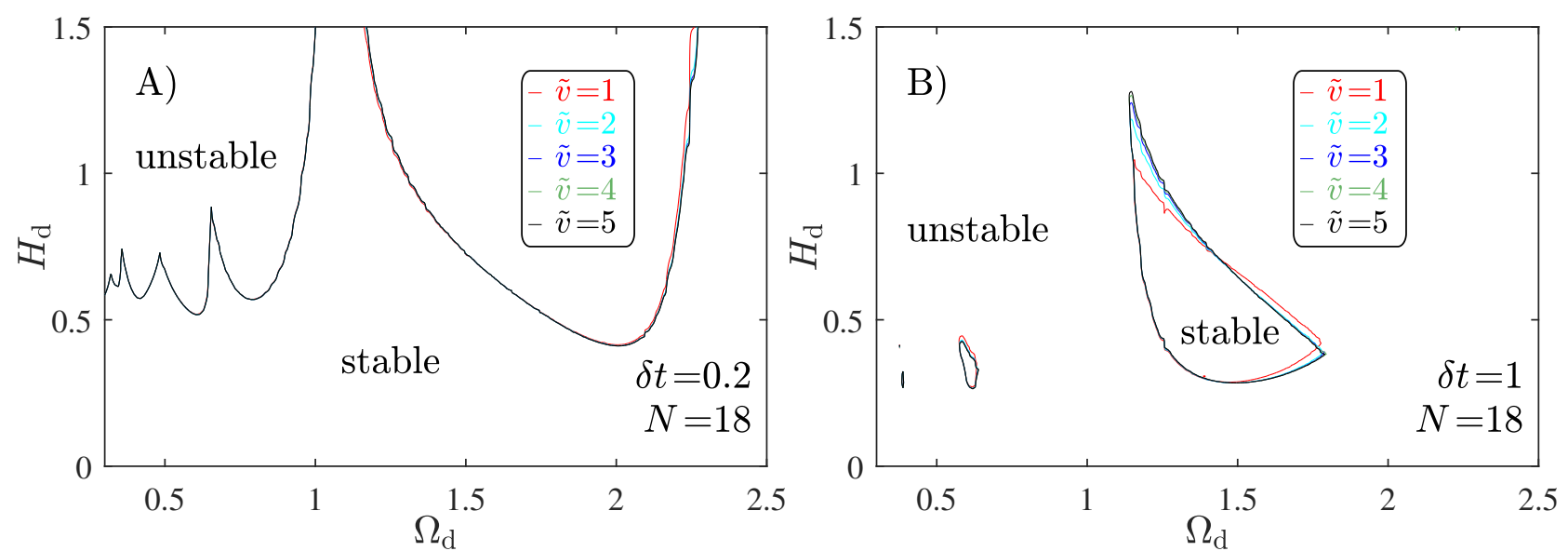

Figure 12: Stability lobes diagrams, computed by the PT method, for down-milling operations with active damper using system parameters given in Table 3 and control parameters $v=1$, $k_{P}=0.2, k_{D}=0.2$. In panels A) and B) stability boundaries are shown for two different $\delta t$ sampling times, with fixed order $N$ of polynomial approximation and increasing resolution $\tilde{v}$. 

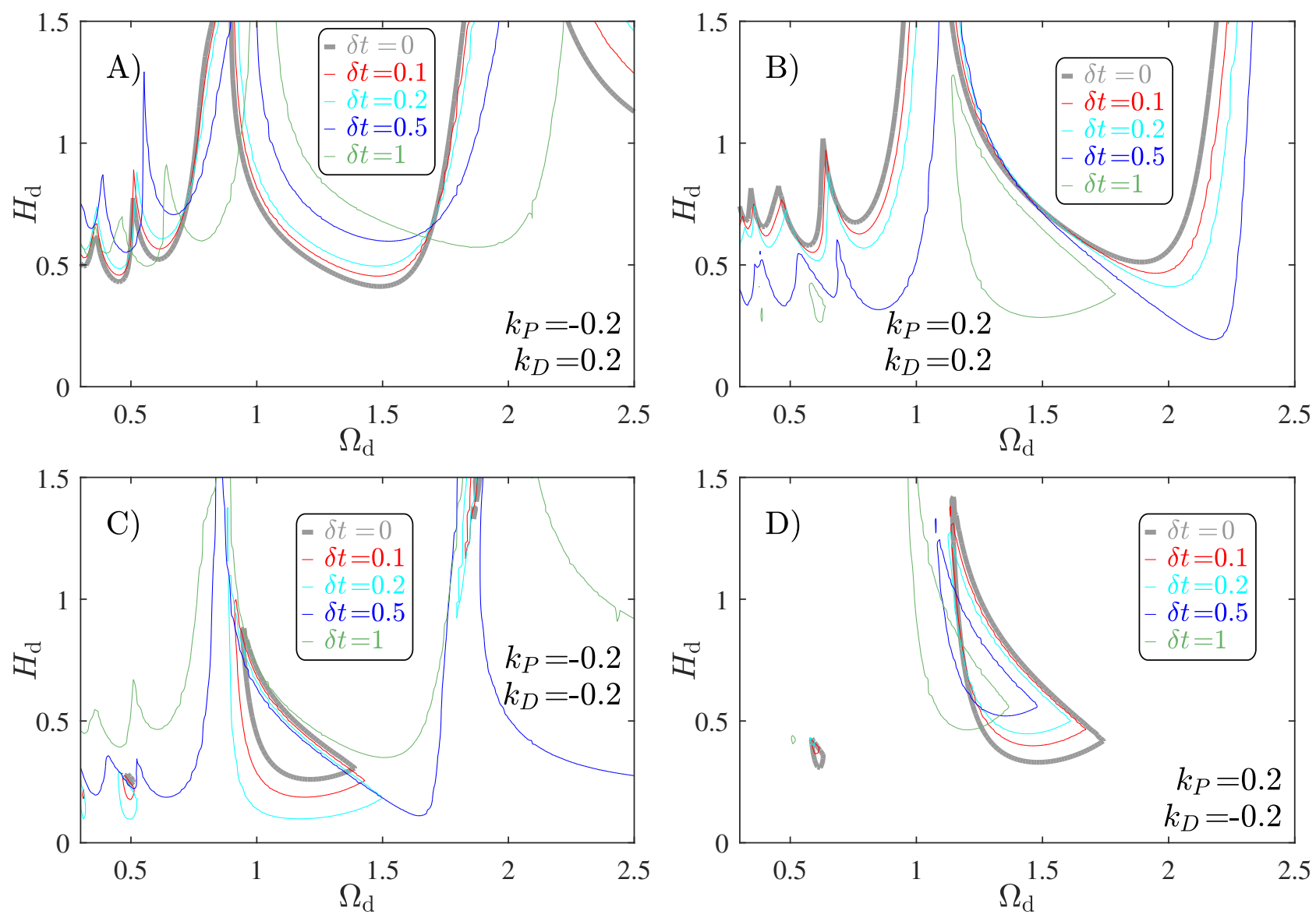

Figure 13: Stability lobes diagrams, computed by the PT method for down-milling operations with active damper using system parameters given in Table 3 . The stability boundaries were computed using order $N=18$ of polynomial approximation and, for non-zero $\delta t$, resolution $\tilde{v}=5$. Panels A)-D) show stability boundaries for different $\left(k_{P}, k_{D}\right)$ control gain combinations with $v=1$ and increasing $\delta t$ sampling times.

numerical schemes presented in Section 3. Similarly as in Section 4.1, the accurate dominant multiplier $\mu_{\mathrm{PT}}^{*}$ for the PT method was computed using $N=80$ and $\tilde{v}=40$, while for the SE method the accurate dominant multiplier $\mu_{\mathrm{SE}}^{*}$ was computed using $N=80$ and $E=1$. As Table 4 and Figure 5 show, the absolute values of dominant multipliers computed by the PT and SE methods converge to the same values in all investigated points of the $\left(\Omega_{\mathrm{d}}, H_{\mathrm{d}}\right)$ parameter plane with a normalized error less than $0.2 \%$. In conclusion, similarly as in Section 4.1, it seems likely that the presented methods give results convergent to the exact stability boundaries and dominant characteristic multipliers of 71 ). This convergence property might hold for arbitrary control and system parameters as well. However, this conjecture can only be verified by a precise theoretical convergence analysis which is out of the scope of this paper. Nevertheless, the above numerical study can serve as a good starting point for such an analysis.

As Section 3 shows, the hybrid terms in DDEs impose additional difficulty for stability computations. It is, therefore, reasonable to ask: whether in real applications of milling processes the inclusion of the hybrid nature of control terms is of significance? In order to answer this question, the boundary of stability was calculated for different control parameter combinations with increasing $\delta t$ sampling time. The results are shown in Figure 13. It can be seen that as the dimensionless sampling time $\delta t=\omega_{\mathrm{n}} \Delta t$ increases, the difference in the stability boundary also increases with respect to the case $\delta t=0$. Note that in machining centers, the dominant natural angular frequency $\omega_{\mathrm{n}}$ of the tool is usually high in order to keep the amplitude of the 
forced vibrations small. This results that the frequency of actuation must be high-enough, in order to neglect the effect of the hybrid terms. On the other hand, it is important to highlight that the hybrid terms in (1) assume that after the arrival of new data, the control force is updated in zero time. In other words, it is assumed that there are no transients in the control force, the response of the actuator is infinitely fast. This can be a reasonable assumption as long as the transients are short compared to the actuation period. However, as the actuation period is decreased, the significance of the transient behavior of the actuator becomes more essential. In conclusion, the hybrid nature of control terms in the governing equations affects stability properties if the lowest dimensionless sampling time is large.

\section{Conclusions}

The spectral element method and pseudospectral tau method has been extended to the stability analysis of a class of time-periodic hybrid time-delay systems involving delayed terms with both continuous and piecewise constant arguments (that is involving continuous and discrete delay terms simultaneously). Both methods give a finite dimensional approximation for the monodromy operator whose dominant characteristic exponent uniquely determines stability. The numerical methods have been applied to two engineering applications: to a coupled haptic system and to a milling process subjected to active vibration suppression via digital feedback control. In these applications both continuous and discrete delay terms have been considered simultaneously. Numerical convergence analysis was carried out and stability boundaries were computed in the plane of control and system parameters, furthermore the convergence of dominant characteristic multipliers was investigated in 6 parameter points of the stability diagrams. When exact results were available, both methods converged to the exact stability boundaries in all investigated cases. Furthermore, when no exact stability boundaries and dominant characteristic multipliers could be computed, the results of both numerical methods converged in all investigated cases to the same stability boundaries and dominant characteristic multipliers. Since the approximation concept of the monodromy operator is fundamentally different for the two numerical methods, it is likely that both methods provide convergent results to the exact boundary of stability and dominant characteristic multipliers. Although only numerical case studies were presented for convergence analysis, these results can give a good starting point for a future theoretical convergence analysis. In addition to the numerical convergence analysis of approximation schemes, the computed results showed that in both engineering applications neglecting the discrete nature in the control terms can lead to significant differences in stability properties. In particular, it was shown for milling processes with active damping that the higher the dimensionless sampling time (normalized by the natural angular frequency of the tool) the more stability boundaries are altered by the discrete nature of the controller. The computed stability diagrams can provide a useful tool in tuning the parameters of hybrid time-delay systems, for example, in the adjustment of control parameters of haptic devices and in the selection of machining parameters of milling processes subjected to active damping.

\section{Acknowledgments}

This work has been supported by the European Research Council under the European Union's Seventh Framework Programme (FP/2007-2013) / ERC Advanced Grant Agreement n. 340889. The authors thank J. Kovecses and L.L. Kovacs for the useful discussion on coupled haptic systems. 


\section{Appendices}

\section{Appendix A Lagrange base polynomials}

The barycentric form of Lagrange polynomials is

$$
\phi_{k}(\zeta)=\frac{\frac{\varpi_{k}}{\zeta-\zeta_{k}}}{\sum_{l=1}^{n+1} \frac{\varpi_{l}}{\zeta-\zeta_{l}}}
$$

where the barycentric weights are

$$
\varpi_{k}=\frac{1}{\omega^{\prime}\left(\zeta_{k}\right)}, \quad \omega(\zeta)=\prod_{k=1}^{n+1}\left(\zeta-\zeta_{k}\right) .
$$

The derivative of Lagrange base polynomials at the points of interpolation is given by

$$
\phi_{k}^{\prime}\left(\zeta_{l}\right)= \begin{cases}\frac{\varpi_{k} / \varpi_{l}}{\zeta_{l}-\zeta_{k}} & k \neq l, \\ -\sum_{\substack{q=1 \\ q \neq k}}^{n+1} \frac{\varpi_{q} / \varpi_{k}}{\zeta_{k}-\zeta_{q}} & k=l .\end{cases}
$$

\section{Appendix B Chebyshev points of second kind}

The Chebyshev points of second kind are

$$
\zeta_{k}=\cos \left(\frac{(k-1) \pi}{n}\right), \quad k=1,2, \ldots, n+1
$$

\section{Appendix C Legendre-Gauss-Lobatto points and weights}

The Legendre-Gauss-Lobatto points $\eta_{q}$ are the roots of $\left(1-\zeta^{2}\right) \psi_{n+1}^{\prime}(\zeta)$, that is $-1,1$ and the roots of the first derivative of the Legendre polynomial of order $n$, defined in Appendix D. The quadrature weights are given by

$$
w_{q}= \begin{cases}\frac{2}{n(n+1)} & q=1, n+1 \\ \frac{2}{n(n+1) \psi_{n+1}^{2}\left(\eta_{q}\right)} & q=2,3, \ldots, n .\end{cases}
$$

\section{Appendix D Legendre polynomials}

According to Bonnet's recursion formula, the Legendre polynomials up to order $n$ are

$$
\begin{aligned}
& \psi_{1}(\zeta)=1 \\
& \psi_{2}(\zeta)=\zeta \\
& \psi_{i}(\zeta)=\frac{2 i-3}{i-1} \zeta \psi_{i-1}(\zeta)-\frac{i-2}{i-1} \psi_{i-2}(\zeta), \quad i=3,4, \ldots, n+1
\end{aligned}
$$




\section{References}

[1] G. Stepan, Modelling nonlinear regenerative effects in metal cutting, Philosophical Transactions of the Royal Society A 359 (2001) 739-757.

[2] K. J. Åström, R. M. Murray, Feedback Systems, Princeton University Press, New Jersey, 2008.

[3] G. Orosz, R. E. Wilson, G. Stepan, Traffic jams: dynamics and control, Philosophical Transactions of the Royal Society A 368 (2010) 4455-4479.

[4] J. Milton, J. L. Cabrera, T. Ohira, S. Tajima, Y. Tonosaki, C. W. Eurich, S. A. Campbell, The time-delayed inverted pendulum: Implications for human balance control, Chaos 19 (026110) (2009) 1-12.

[5] Y. Kuang, Delay differential equations with applications in population dynamics, Academic Press, New York, 1993.

[6] G. Rost, J. Wu, SEIR epidemiological model with varying infectivity and infinite delay, Mathematical Biosciences and Engineering 5 (2) (2008) 389-402.

[7] G. Stepan, Retarded dynamical systems, Longman, Horlow, 1989.

[8] T. Insperger, G. Stepan, Semi-discretization for time-delay systems, Springer, New York, 2011.

[9] Y. Ding, L. M. Zhu, X. J. Zhang, H. Ding, A full-discretization method for prediction of milling stability, International Journal of Machine Tools and Manufacture 50 (2010) 502-509.

[10] F. A. Khasawneh, B. P. Mann, A spectral element approach for the stability analysis of time-periodic delay equations with multiple delays, Communications in Nonlinear Science and Numerical Simulation 18 (2013) 2129-2141.

[11] D. Breda, S. Maset, R. Vermiglio, Stability of Linear Delay Differential Equations: A Numerical Approach with MATLAB, Springer, New York, 2015.

[12] E. A. Butcher, O. A. Bobrenkov, On the Chebyshev spectral continuous time approximation for constant and periodic delay differential equations, Communications in Nonlinear Science and Numerical Simulation 16 (2011) 1541-1554.

[13] G. Stepan, Vibrations of machines subjected to digital force control, International Journal of Solids and Structures 38 (2001) 2149-2159.

[14] S. Shayan-Amin, L. L. Kovacs, J. Kovecses, The role of mechanical properties on the behaviour and performance of multi-DoF haptic devices, in: Proceedings of the IEEE World Haptics Conference, Daejeon, Korea, 2013, pp. 725-730.

[15] L. L. Kovacs, J. Kovecses, Dynamics of coupled haptic systems, in: Proceedings of the IEEE World Haptics Conference, Evanston, USA, 2015, pp. 286-292.

[16] T. Insperger, D. Lehotzky, G. Stepan, Regenerative delay, parametric forcing and machine tool chatter: A review, IFAC-PapersOnLine 48(12) (2015) 322-327. 
[17] T. G. Molnar, T. Insperger, On the robust stabilizability of unstable systems with feedback delay by finite spectrum assignment, Journal of Vibration and Control 22(3) (2014) 649661.

[18] D. Hajdu, T. Insperger, Demonstration of the sensitivity of the smith predictor to parameter uncertainties using stability diagrams, International Journal of Dynamics and Control 4 (2016) 384-392.

[19] T. Insperger, Act-and-wait concept for continuous-time control systems with feedback delay, IEEE Transactions on Control Systems Technology 14(5) (2006) 974-977.

[20] J. I. Ge, , G. Orosz, Optimal control of connected vehicle systems with communication delay and driver reaction time, IEEE Transactions on Intelligent Transportation Systems 18(8) (2017) 2056-2070.

[21] D. Lehotzky, T. Insperger, G. Stepan, Extension of the spectral element method for stability analysis of time-periodic delay-differential equations with multiple and distributed delays, Communications in Nonlinear Science and Numerical Simulation 35 (2016) 177189.

[22] J. K. Hale, S. M. V. Lunel, Introduction to functional differential equations, SpringerVerlag, New York, 1993.

[23] D. Lehotzky, T. Insperger, A pseudospectral tau approximation for time delay systems and its comparison with other weighted-residual-type methods, International Journal for Numerical Methods in Engineering 108 (2016) 588-613.

[24] F. L. Trefethen, Is Causs quadrature better than Clenshaw-Curtis?, SIAM Review 50(1) (2008) 67-87.

[25] L. L. Kovacs, J. Kovecses, G. Stepan, Analysis of effects of differential gain on dynamic stability of digital force control, International Journal of Non-Linear Mechanics 43 (2008) $514-520$.

[26] Y. Altintas, Manufacturing Automation, Cambridge University Press, New York, 2012.

[27] Y. S. Tarng, J. Y. Kao, E. C. Lee, Chatter suppression in turning operations with a tuned vibration absorber, Journal of Materials Processing Technology 105 (2000) 55-60.

[28] N. D. Sims, Vibration absorbers for chatter suppression: A new analytical tuning methodology, Journal of Sound and Vibration 301 (2007) 592-607.

[29] D. Mei, T. Kong, A. J. Shih, Z. Chen, Magnetorheological fluid-controlled boring bar for chatter suppression, Journal of Materials Processing Technology 209 (2009) 1861-1870.

[30] M. Zatarain, I. Bediaga, J. Munoa, R. Lizarralde, Stability of milling processes with continuous spindle speed variation: Analysis in the frequency and time domains, and experimental correlation, CIRP Annals - Manufacturing Technology 57 (2008) 379-384.

[31] N. J. M. van Dijk, N. van de Wouw, E. J. J. Doppenberg, H. A. J. Oosterling, H. Nijmeijer, Robust active chatter control in the high-speed milling process, IEEE Transactions on Control Systems Technology 20(4) (2012) 901-917.

[32] J. Munoa, I. Mancisidor, N. Loix, L. G. Uriarte, R. Barcena, M. Zatarain, Chatter suppression in ram type travelling column milling machines using a biaxial inertial actuator, CIRP Annals - Manufacturing Technology 62 (2013) 407-410. 
[33] D. Lehotzky, T. Insperger, Stability of turning processes subjected to digital PD control, Periodica Polytechnica Mechanical Engineering 56 (1) (2012) 33-42.

[34] D. Lehotzky, T. Insperger, Stabilizability diagram for turning processes subjected to digital PD control, International Journal of Dynamics and Control 2 (2014) 46-54.

[35] F. W. Taylor, On the art of cutting metals, Transaction of ASME 28 (1907) 31-350.

[36] D. Lehotzky, T. Insperger, F. Khasawneh, G. Stepan, Spectral element method for stability analysis of milling processes with discontinuous time-periodicity, International Journal of Advanced Manufacturing Technology 89(9) (2017) 2503-2514. 